\title{
Contributions of Pitch Contour, Tonality, Rhythm, and Meter to Melodic Similarity
}

\author{
Jon B. Prince \\ Murdoch University
}

\begin{abstract}
The identity of a melody resides in its sequence of pitches and durations, both of which exhibit surface details as well as structural properties. In this study, pitch contour (pattern of ups and downs) served as pitch surface information, and tonality (musical key) as pitch structure; in the temporal dimension, surface information was the ordinal duration ratios of adjacent notes (rhythm), and meter (beat, or pulse) comprised the structure. Factorially manipulating the preservation or alteration of all of these forms of information in 17 novel melodies (typifying Western music) enabled measuring their effect on perceived melodic similarity. In Experiment 1,34 participants (varied musical training) rated the perceived similarity of melody pairs transposed to new starting pitches. Rhythm was the largest contributor to perceived similarity, then contour, meter, and tonality. Experiment 2 used the same melodies but varied the tempo within a pair, and added a prefix of 3 chords, which oriented the listener to the starting pitch and tempo before the melody began. Now contour was the strongest influence on similarity ratings, followed by tonality, and then rhythm; meter was not significant. Overall, surface features influenced perceived similarity more than structural, but both had observable effects. The primary theoretical advances in melodic similarity research are that (a) the relative emphasis on pitch and temporal factors is flexible; (b) pitch and time functioned independently when factorially manipulated, regardless of which dimension is more influential; and (c) interactions between surface and structural information were unreliable and never occurred between dimensions.
\end{abstract}

Keywords: pitch, time, music, similarity

An enduring question in human perception is what makes two melodies sound similar. In fact, music is an especially well-suited domain for examining the general concept of similarity, as it consists of clearly delineated dimensions that not only exhibit hierarchical structure and statistical regularities, but can be manipulated independently while preserving the naturalistic properties of the stimulus. Although music has multiple dimensions, pitch and time have received the most attention-likely because, for the overwhelming majority of music, they together define the identity of a musical piece and exhibit the greatest degree of complexity. This complexity makes it difficult to sort out the details of how all the components of pitch and time contribute to perceived similarity. Indeed, how pitch and time combine in music perception remains an open question (for reviews, see Prince, Thompson, \& Schmuckler, 2009; Schellenberg, Stalinski, \& Marks, 2014).

Pitch and time have critical information at both the superficial surface level and at deeper structural levels (Krumhansl, 2000). The aim of this article is to examine how surface and structural information in both pitch and time affect perceived melodic similarity, and in particular, how they combine. For the purposes of this article, surface

Thanks to Rachael Davey, Scott Devenish, Andrew Mannucci, Sandra O'Shea, and Taryn van Gramberg for help with Experiment 1 data collection.

Correspondence concerning this article should be addressed to Jon Prince, School of Psychology and Exercise Science, Murdoch University, 90 South Street, Murdoch WA 6150, Australia. E-mail: j.prince@murdoch.edu.au information refers to pitch contour and rhythm, as they are comprised of information directly available at the level of the musical surface. ${ }^{1}$ The structural information in this case is tonality and meter, as they represent information derived from the surface.

Pitch contour refers to the pattern of ascending and descending pitch intervals of a melody, and it is a primary component of melodic perception (for reviews, see Deutsch, 2013; Schmuckler, 2009). Dowling (1978) presents contour as one of two critical factors (tonality being the other) in melodic perception and memory, showing that a nonexact imitation of the standard melody is often confused as a match when it has a similar contour. Indeed, even when wildly out of tune, singers preserve the general contour of a melody (Pfordresher \& Mantell, 2014). The importance of contour is also evidenced by its early emergence-infants as young as 5 months differentiate melodies primarily on the basis of their contour (for a review, see Trehub \& Hannon, 2006).

The other component to Dowling's (1978) model of melodic perception is based on tonality (musical key), which refers to the hierarchical organization of the 12 unique pitch classes per octave used in Western music, arranged around a central reference pitch, or tonic. For instance, in the key of $\mathrm{G}$ major, the pitch class $\mathrm{G}$ is the tonic-it is the most psychologically stable pitch and central cognitive reference point; all other pitches are ordered in a hier-

\footnotetext{
${ }^{1}$ Contour and rhythm can also be considered as structural, as changing either of them may also change the identity of the melodic sequence. In the present article, these will be considered surface characteristics, if only to differentiate them from the more clearly structural variables of tonality and meter.
} 
archical fashion relative to the tonic. Tonics are heard more frequently, make better endings for melodies, and confer processing benefits (Krumhansl, 1990). Tonality is a fundamental characteristic of music, functioning as a structure on which to encode additional information (Dowling, 1978), and therefore is a strong contributor to melodic processing (for a review, see Krumhansl \& Cuddy, 2010). Although some methodologies show musicians as more sensitive to tonality than untrained listeners (Krumhansl \& Shepard, 1979), tonality strongly influences music perception regardless of expertise (Bigand \& Poulin-Charronnat, 2006), even for tone-deaf individuals (Tillmann, Gosselin, Bigand, \& Peretz, 2012). Looking more generally than the music cognition literature, physics experts tend to emphasize structural information in problem categorization at the expense of surface, relative to novices (Chi, Feltovich, \& Glaser, 1981).

Pitch cannot function alone in music-it is structured in time. Patterns of duration and relative timing comprise the rhythm, or temporal surface information, which has a strong role in melodic processing (for a review, see McAuley, 2010). Although any rhythmic change will decrease melodic recognition, not all aspects are equally influential. For example, Schulkind (1999) found that preserving the relative pattern of short and long notes (i.e., rhythm) while changing their absolute ratios (e.g., changing $.2 \mathrm{~s}, .6 \mathrm{~s}, .3 \mathrm{~s}$ to $.1 \mathrm{~s}, .6 \mathrm{~s}, .4 \mathrm{~s}$ ) impaired recognition less than reordering the original durations (e.g., .6 s, .2 s, .3 s).

Repeating patterns in rhythmic sequences leads to the abstraction of an underlying metrical pulse (beat), or meter (Lerdahl \& Jackendoff, 1983). This hierarchical temporal structure (Palmer \& Krumhansl, 1990) guides our attention (Jones \& Boltz, 1989), improves the processing of events that coincide with the pulse (Barnes \& Jones, 2000), modulates our interpretation of ambiguous rhythmic sequences (Desain \& Honing, 2003), and influences perceived melodic similarity (Eerola, Järvinen, Louhivuori, \& Toiviainen, 2001). There is also a prodigious literature on the role of meter in sensorimotor synchronization (for reviews, see Repp, 2005; Repp \& Su, 2013).

To perceive a melody, the listener must integrate the surface and structural information in both pitch and time, but how this occurs is unclear, particularly with regard to independence or interaction. In the case of contour and tonality (both pitch variables), they are theoretically independent, in that any number of different pitch sequences can establish a tonal center (musical key). Of course, the exact choice of pitch classes will determine whether the sequence is tonal, but the general up-down shape of the pitch profile does not restrict its tonality.

Accordingly, the majority of experimental evidence suggests that contour and tonality are processed and function independently (Dowling, Kwak, \& Andrews, 1995; Edworthy, 1985; Eiting, 1984; Trainor, McDonald, \& Alain, 2002). Dowling and colleagues have established that when comparing novel melodies with no delay, listeners primarily rely on contour-they are likely to falsely recognize a melody in the same key as a match if it has a similar contour. But for longer delays with interspersed melodies, listeners abstract a more detailed representation of the melody that is key-invariant and is more sensitive to structural information (Dewitt \& Crowder, 1986; Dowling, 1978; Dowling et al., 1995). This differential contribution of tonality and contour to melodic memory implies independence of function. Repeated listenings also result in more observable effects of structural features on melodic perception, such as tonality (Pollard-Gott, 1983; Serafine, Glassman, \& Overbeeke, 1989). Further, when sequences are atonal (not conforming to any musical key), listeners primarily rely on contour for processing melodies (Freedman, 1999; Krumhansl, 1991), also consistent with independence.

However, there is contrary evidence, such as findings that tonality only matters when the contour information is preserved-without a matching contour, violating tonality had no effect on melody recognition (Massaro, Kallman, \& Kelly, 1980). Additionally, the exact arrangement of intervals in three-note sequences can influence the ease of establishing tonality (Cuddy \& Cohen, 1976). Interestingly, the reverse pattern has also been reported-where processing contour information is easier for tonal melodies (Bartlett \& Dowling, 1988; Cuddy, Cohen, \& Mewhort, 1981; Dowling, 1991). Thus, tonality and contour may not be fully independent.

For rhythm and meter (both temporal variables), it is again the case that any number of different surface (rhythmic) patterns may instantiate a given structure (meter), suggesting some degree of theoretical independence. Although the particular sequence of time intervals between events determines whether a metrical framework can be extracted from a rhythmic pattern, the ordinal sequence itself does not necessarily constrain its potential metrical interpretations. However, the exact sequence of intervals is not trivial—rhythmic patterns are a primary factor in establishing the perception of musical events, such that the occasional long gap between events in a sequence indicates a grouping boundary (Garner \& Gottwald, 1968). Interonset intervals that are related by regular simple integer ratios (e.g., 1:2, 1:3) can go on to establish metrical frameworks (Povel \& Essens, 1985), but even those with complex ratios (e.g., 1:2.5, 1:3.5) can successfully form into groups and be processed with (admittedly lower) accuracy (Essens, 1986; Essens \& Povel, 1985; Handel \& Oshinsky, 1981), as well as learned implicitly (Schultz, Stevens, Keller, \& Tillmann, 2013). Thus, the sequence of durations in a rhythmic pattern has unique importance beyond its role in establishing a meter (Monahan, Kendall, \& Carterette, 1987). Nevertheless, it is unlikely that rhythm and meter can function entirely independently — not only is meter extracted from the rhythmic surface, but the metric framework can modify perception of rhythmic sequences (Desain \& Honing, 2003).

What about cross-dimensional relations in surface and structure? For instance, can tonality affect rhythm perception, or can meter affect contour perception? This question is even more difficult to answer, partly because the relation between pitch and timing information varies greatly depending on the stimuli and task (Barnes \& Johnston, 2010; Prince, 2011; Tillmann \& Lebrun-Guillaud, 2006). Melodic recognition accuracy decreases when the standard and comparison melodies have different rhythmic groupings (Dowling, 1973; Jones \& Ralston, 1991) or metrical frameworks (Acevedo, Temperley, \& Pfordresher, 2014). Increasing the tempo of interleaved melodies fosters their segregation into separate streams, although this requires alternations between low and high pitches at extremely rapid rates of less than 150 ms between tones (Bregman, 1990). The exact combination of rhythmic and melodic patterns can also influence the ability to discriminate targets and decoys (Jones, Summerell, \& Marshburn, 1987), although, in that study, listeners only used rhythmic patterns to differentiate melodies if the decoy contour remained the same. Boltz (2011) found that raising the pitch or brightening the timbre of melodies makes them seem faster. Using trained musicians only, Abe and Okada (2004) reported that shifting the phase of pitch and temporal patterns (by one to two positions) altered the interpretation 
of the musical key, but not the perceived meter, providing evidence for an asymmetric relationship between meter and tonality. However, other research found the opposite asymmetry, in which musicians were more likely to report that probes following a melody were on the beat if the pitch was tonally stable, but pitch judgments were unaffected by their metrical position (Prince, Thompson, et al., 2009).

Research on the relative contribution of pitch and time to perceived similarity of novel melodies generally finds that temporal surface information is most prominent. Halpern (1984; Halpern, Bartlett, \& Dowling, 1998) analyzed the similarity ratings of 16 melodic sequences, and found that changes to the rhythmic properties were most influential on ratings, followed by contour, and then whether the melody was in a major or minor key (tonal structure). Rosner and Meyer (1986) also reported that rhythm (the temporal surface) was the most important factor on similarity of 12 melodies, followed by a mixture of surface and structural pitch variables.

Using qualitative descriptions of nine extracts from two musical pieces, Lamont and Dibben (2001) highlighted the role of surface features such as dynamics (loudness) and tempo over pitch height and contour. Moreover, these authors found no role of deeper structural information. McAdams, Vieillard, Houix, and Reynolds (2004) asked listeners to group 34 sections of a single musical piece according to their own subjective criteria (i.e., no predefined categories), and then provide terms that capture the essence of what makes a group similar. Temporal surface (tempo and rhythm) descriptors were the most prevalent and dominant characteristics, over pitch surface variables (average pitch height, contour).

Eerola et al. (2001) predicted the perceived musical similarity of 15 folk melodies based on statistical properties (frequency-based surface information) and descriptive characteristics (akin to structural information). The descriptive variables accounted for more variance than the statistical ones, but the best solution came from a combination of both variable types. They acknowledged the possibility of overfitting the data, but it is nonetheless important that both forms of information can contribute uniquely to perceived similarity.

None of the studies discussed previously directly address the relationship between pitch and temporal information in melodic similarity beyond their relative contribution - that is, how might they affect one another? In fact, there is only one article that touches on this issue (Monahan \& Carterette, 1985). These authors found that five dimensions best explained similarity ratings of 32 melodies; the first three reflected temporal characteristics, and the last two were pitch-based. But the most immediately relevant result to cross-dimensional relations was an individual-differences tradeoff between reliance on pitch and temporal information-participants who placed strong weight on temporal factors de-emphasized the pitch factors, and vice versa.

The question of interactions between the parameters of contour, rhythm, tonality, and meter requires a delicate balance between methodical experimental control and natural musical context. Because listeners in McAdams et al.'s (2004) study established their own subjective criteria, it is difficult to establish quantitative interpretations of the data, and moreover, the attributes covaried-as would be expected in normal music heard in more naturalistic conditions. Rosner and Meyer (1986) stated that there should be interactions between them, but were not able to directly assess this possibility. Similarly, when explaining the relative lack of explanatory value of some of their measured variables, Eerola et al. (2001) pointed to the fact that their melodies varied simultaneously on multiple dimensions, and they were using an "oversimplified representation" of the melodies in their analyses. They recommended that future research vary the stimuli in a more systematic and controlled manner to more exactly assess their relative contribution.

\section{Experiment 1}

As stated earlier, the main goal of the present research is to examine how surface and structural information in the dimensions of both pitch and time combine in contributing to melodic similarity. On the basis of the background literature, it is proposed that (a) surface information should be more influential than structure for novel melodies, and (b) temporal manipulations should have greater effect than pitch. However, the primary theoretical question is to test for interactions between these variables (contour, rhythm, tonality, and meter), not just their respective roles. Because the background literature provides no clear guidance on this issue, the present research approaches this issue methodically by using a factorial manipulation of all these variables. Additionally, a much larger stimulus set than typically employed was created, using 17 typical melodies as starting points for creating 16 variants that factorially preserved (or altered) the contour, rhythm, tonality, and meter of the original melody (giving 272 unique sequences). Accordingly, no listener heard a given sequence twice, greatly reducing the potential role of learning during the experimental session affecting similarity judgments. Three analysis techniques were employed, including categorical ANOVA analyses (made possible by the factorial design), linear regression with nonintercorrelated predictors, and factor analysis. Together, this approach is intended to provide a close quantitative examination of the roles of contour, rhythm, tonality, and meter in melodic similarity, and, in particular, how they combine.

\section{Method}

Participants. There were 34 participants, with an average age of 22.6 years $(S D=4.7)$ and 3.5 years of musical training $(S D=$ 4.8). Participants were recruited from the Murdoch University community, largely undergraduate psychology students. Compensation was either course credit or $\$ 10$.

Stimuli. There were 17 normal melodies $(M$ length $=12.1$ notes, $4.8 \mathrm{~s}$ ) that served as original seed melodies from which all 16 variants were created. The seed melodies were all in "common time" (four beats per measure); 12 used the major scale and five used the melodic minor scale. The pitch and temporal characteristics of the seed melodies were varied independently, in factorial fashion. Table 1 summarizes the manipulation levels and their properties. There were four levels of pitch manipulation, where the first level (p1) was the original pitch sequence, that is, unaltered from the original melody. The melodies strongly established a musical key, as assessed by the Krumhansl-Schmuckler keyfinding algorithm (Krumhans1, 1990; Krumhansl \& Schmuckler, 1986) - the average correlation coefficient of the distribution of pitches in $\mathrm{p} 1$ sequences with the intended key was $.84(S D=.08)$. This coefficient is known as the maximum key correlation (MKC).

The $\mathrm{p} 2$ level preserved the global pitch contour of its corresponding seed melody, but had a different set of pitches in order to destroy the sense of musical key (i.e., they did not fit in any Western major or minor key). The artificial set of pitches (or scale) consisted of A B C\# D D\# F G; like other scales, it could be transposed to start on any pitch. This scale preserves important characteristics of musical scales 
Table 1

Explanation of Pitch and Time Manipulation Levels

\begin{tabular}{|c|c|c|c|c|}
\hline Level & Name & Description & Surface preserved? & Structure preserved? \\
\hline p1 & Pitch original & Unaltered (original) sequence of pitches & Yes & Yes \\
\hline $\mathrm{p} 2$ & Atonal original contour & $\begin{array}{l}\text { Pitches replaced with artificial scale (A B C\# D D\# F G), but } \\
\text { retaining contour }\end{array}$ & Yes & No \\
\hline p3 & Contour-violated & $\begin{array}{l}\text { Randomly shuffled order of original pitches, not violating } \\
\text { tonality }\end{array}$ & No & Yes \\
\hline $\mathrm{p} 4$ & Contour-violated-atonal & Randomly shuffled order of $\mathrm{p} 2$ pitches & No & No \\
\hline $\mathrm{t} 1$ & Time original & Unaltered (original) sequence of durations & Yes & Yes \\
\hline $\mathrm{t} 2$ & Ametric original rhythm & $\begin{array}{l}\text { Durations changed to non-metric }(200,280,530,650 \mathrm{~ms}) \text {, but } \\
\text { preserving ordinal scaling (rhythm) }\end{array}$ & Yes & No \\
\hline $\mathrm{t} 3$ & Rhythm-violated & $\begin{array}{l}\text { Randomly shuffled order of original durations, unchanged } \\
\text { meter }\end{array}$ & No & Yes \\
\hline $\mathrm{t} 4$ & Rhythm-violated-ametric & Randomly shuffled order of t 2 durations & No & No \\
\hline
\end{tabular}

Note. The first and last pitches, as well as first and last durations, were unchanged in all conditions.

(Trehub, Schellenberg, \& Kamenetsky, 1999), in that it used 7 of 12 pitch classes per octave, neighboring pitches were either one or two semitones apart (one semitone is the smallest possible step in Western music), and not all steps were equally sized. The p2 level therefore corresponded to a preservation of surface (contour) but violation of structure (tonality). Comparing the contour of corresponding $\mathrm{p} 1$ and p2 sequences by converting their notes to a series of pitch heights (e.g., 1, 4, 3, 6, and 2, 4, 3, 5) and correlating them resulted in a high level of agreement $(M r=.93, S D=.06)$. Conversely, the average $\mathrm{MKC}$ of $\mathrm{p} 2$ sequences was low $(M \mathrm{MKC}=.44, S D=.11)$ compared with the much higher average $\mathrm{p} 1 \mathrm{MKC}$. The first and last notes of the sequence were unchanged from the seed melody, which were also members of the artificial scale.

A contour-violated manipulation level (p3) pseudorandomly shuffled the order of the seed melody pitches, but did not add or delete any pitches. The randomization had the constraints that the first and last pitch had to stay the same as the seed melody, but no other note could remain in its original place. This change therefore retained the tonality (structure) of the seed melody ( $M$ MKC $=.83, S D=.09$ ), while disrupting its contour (surface), as the average correlation of p3 and p1 pitch sequences was $r=.14(S D=.34)$.

The final pitch manipulation level (p4) was a contour-violatedatonal variant created by pseudorandomly shuffling the order of the atonal p2 level, thereby destroying both the surface and structure of the seed melody. The randomization constraints were the same as those used for creating the $\mathrm{p} 3$ level from the $\mathrm{p} 1$ level, but instead were applied to the $\mathrm{p} 2$ level. The average MKC of the $\mathrm{p} 4$ sequences was $.50(S D=.12)$, and the average correlation of $\mathrm{p} 3$ and $\mathrm{p} 4$ pitch sequences was .04 $(S D=.35)$.

The four levels of time manipulation were also factorial variations of surface and structure. There were no silent gaps between notes for all levels, so durations were equivalent to interonset intervals. The t1 level was the original sequence of durations, which, for each seed melody, had four unique duration values: $167 \mathrm{~ms}$ (eighth note), 333 $\mathrm{ms}$ (quarter note), $500 \mathrm{~ms}$ (dotted eighth note), or $667 \mathrm{~ms}$ (half note). All t1 levels had a regular beat and were clearly metric, as measured by comparing the distribution of note onsets with the idealized metric hierarchy of Palmer and Krumhansl (1990); the average correlation was $.78(S D=.05)$.

The $\mathrm{t} 2$ level was an ametric variant that preserved the rhythmic pattern of the seed melody. This manipulation was accomplished by changing each of the four regular note durations used in the $\mathrm{t} 1$ level to a matched nearby value $(200,280,530$, and $650 \mathrm{~ms}$, respectively). These new durations preserved the surface pattern of relative short and long durations (i.e., rhythm), but destroyed the temporal structure (meter). Whereas the original durations are related by simple integer ratios $(1: 2,1: 3,2: 3)$ that establish a regular beat, the new durations used complex integer ratios (e.g., 5:7, 20:53, 4:13) that did not accommodate any regular metric framework, thus violating the temporal structure. The average correlation of the series of durations comprising the rhythm of $\mathrm{t} 1$ and $\mathrm{t} 2$ sequences was $.98(S D=.01)$, demonstrating excellent preservation of the temporal surface.

The $\mathrm{t} 3$ level pseudorandomly shuffled the order of the seed melody durations, thus creating a rhythm-violated sequence that preserved the metrical structure of the melody, in that all durations still accommodated a regular metrical framework. The randomization had the constraints that the first and last duration had to stay the same as the seed melody, but no other duration could remain in its original place. Retaining the same quantized durations was largely successful in preserving the metrical framework, although the randomization of duration order did result in a weaker correlation with the Palmer and Krumhansl (1990) hierarchy $(M=.63, S D=.14)$. The surface information was demonstrably altered, as the average t1-t3 duration sequence correlation was $.16(S D=.27)$.

The final time manipulation level (t4) violated both the rhythm and the metrical framework of the seed melody, by pseudorandomly shuffling the order of the $\mathrm{t} 2$ durations, using the same constraints as those for generating the $\mathrm{t} 3$ level. The average correlation of $\mathrm{t} 3$ and $\mathrm{t} 4$ duration sequences was $.12(S D=.20)$.

Combining all four pitch levels with four time levels generated 16 variants of each of the 17 seed melodies (see Table 2). Figure 1 depicts some example variants from one seed melody. In a given trial, participants heard two sequences, consisting of two variants of the same seed melody (e.g., p1t4 and p2t3), and judged their similarity. That is, both melodies in a trial were derived from the same seed melody, never different seeds. Regardless of pitch manipulation level, the second melody of a pair always started on a different pitch (i.e., transposed to a new key), in order to avoid a confound between the manipulations of interest and the number of pitches shared between sequences, which affects perceived similarity (van Egmond \& Povel, 1996; van Egmond, Povel, \& Maris, 1996). A tonal sequence and an atonal sequence must have 
Table 2

Manipulation Levels in Pitch and Time, and Resulting Condition Names

\begin{tabular}{lcccc}
\hline & Pitch original & $\begin{array}{c}\text { Atonal original } \\
\text { contour }\end{array}$ & Contour-violated & Contour-violated-atonal \\
\hline Time original & $\mathrm{p} 1 \mathrm{t} 1$ & $\mathrm{p} 2 \mathrm{t} 1$ & $\mathrm{p} 3 \mathrm{t} 1$ & $\mathrm{p} 4 \mathrm{t} 1$ \\
Ametric original rhythm & $\mathrm{p} 1 \mathrm{t} 2$ & $\mathrm{p} 2 \mathrm{t} 2$ & $\mathrm{p} 3 \mathrm{t} 2$ & $\mathrm{p} 4 \mathrm{t} 2$ \\
Rhythm-violated & $\mathrm{p} 1 \mathrm{t} 3$ & $\mathrm{p} 2 \mathrm{t} 3$ & $\mathrm{p} 3 \mathrm{t} 3$ & $\mathrm{p} 4 \mathrm{t} 3$ \\
Rhythm-violated-ametric & $\mathrm{p} 1 \mathrm{t} 4$ & $\mathrm{p} 2 \mathrm{t} 4$ & $\mathrm{p} 3 \mathrm{t} 4$ & $\mathrm{p} 4 \mathrm{t} 4$ \\
\hline
\end{tabular}

mostly different pitch classes because the scale has changed. Thus, comparisons between two tonal sequences should share a similar number of pitch classes as a tonal-atonal pair, in order to separate the effects of pitch class overlap from tonality on perceived similarity. Transposing the melodies to different keys met this need, providing a way to control the number of shared pitch classes between sequences, thus preventing a confound between the manipulations of structure and surface.

Four different starting pitches were used for tonal sequences $(\mathrm{C}$, $\mathrm{D}, \mathrm{E}$, and $\mathrm{G \#}$ ), and a different four were used for atonal sequences (C, C\#, D\#, G\#). The assignment of starting pitches was arranged such that the average number of shared pitch classes ranged between 3.4 and 4.3. Tempo remained constant throughout the experiment in order to control the effects of elapsed time between standard and comparison on the memory trace. Experiment 2 returns to this issue. Melodies were generated as MIDI files in MATLAB and then converted to .wav files, all using the same piano timbre soundfont.

Comparing the 16 different variants provided $16 \times 16=256$ possible combinations for each seed melody (counting both orders of a given pair). Having all participants rate each combination would have made the experimental session too long. Instead, participants only heard one order of each variant combination (e.g., p2t3-p1t1 or p1t1-p2t3), giving 136 trials including match conditions such as $\mathrm{p} 3 \mathrm{t} 4-\mathrm{p} 3 \mathrm{t} 4$. The session took an average of $31 \mathrm{~min}$ to complete. Order combination was counterbalanced, sampling equally from above and below the diagonal of the $16 \times 16$ matrix for each participant. Also counterbalanced across participant was the assignment of melodies and variants, such that each participant never heard the same variant of a given melody more than once. Although a given trial consisted of variants derived from the same seed melody, subsequent trials would be based on a different seed melody.

Procedure. Participants gave informed consent and completed a background questionnaire on musical experience. The experimenter explained the task of rating melodic similarity, and also the concept of transposition by explaining that singing "Happy Birthday" starting on a low note or a high note did not change the melody. That is, it was the pattern of pitches that was important, not the absolute frequencies themselves.

Each trial began by the participant pressing the space bar, after which the first sequence of the pair began. They then had to press the " $\mathrm{s}$ " key to hear the second sequence. This procedure ensured that the participants were aware of the separation between sequences, and was intended to eliminate confusion about when the first sequence ended and the second began. On average, participants waited $0.90 \mathrm{~s}(S D=.42$, median $=0.77)$ between sequences. Immediately following the second sequence, participants were prompted to provide a rating of similarity on the scale of 1 (not at all similar) to 7 (very similar).

Participants completed three practice trials before beginning the full experiment. The first practice trial presented a p1t1-p1t1 combination - an exact transposition of an original seed melody. If they gave a similarity rating below 6 (suggesting confusion regarding the transposition of the second sequence), the experimenter explained that this case was indeed an exact match, that is, both sequences had the same pattern of intervals and durations, despite starting on different pitches, and that this was as similar as the sequences could get. Further, the experimenter reexplained the concept of transposition to ensure that the participant understood the task fully. The remaining practice trials consisted of a p3t1p3t4 and a p1t2-p2t2 pair (randomizing both within-trial sequence order and between-trial pair order); no further instructions regarding a "correct" rating were provided.

Data analysis. Before the main rating data analyses, there were preliminary inspections comprised of manipulation checks, examination of effects of variant order combination, and testing for expertise effects. Subsequently, an ANOVA tested the role of change (i.e., same or different within a trial) of contour, rhythm, tonality, and meter in a categorical analysis (thus a $2 \times 2 \times 2 \times$ 2 univariate equation), made possible by the factorial design of the experiment. This analysis collapsed across participant (after first ensuring decent interparticipant agreement), ${ }^{2}$ and enabled systematic evaluation of the interaction or independence of all manipulated variables.

The second approach was a linear regression equation (following Eerola et al., 2001) predicting the perceived similarity ratings averaged across participant, using continuous objective measures of contour, rhythm, tonality, and meter. The contour predictor was the average correlation coefficient of the two melodies, when coded as a numerical series of pitches ${ }^{3}$; the rhythm predictor was the average correlation of the sequence of durations (ms). Higher coefficients indicate greater predicted surface similarity; thus, positive correlations between these variables and similarity ratings were expected. The tonality predictor was the average absolute difference in tonality (MKC) between the two melodies; the meter

\footnotetext{
${ }^{2}$ Because each participant provided 136 ratings (not the full $16 \times 16$ grid), a repeated-measures ANOVA approach would have resulted in an unacceptably high number of missing cells.

${ }^{3}$ Using the Fourier analysis model of melodic contour (Schmuckler, 1999, 2010), two other measures of contour similarity were tested: the absolute difference score between the amplitude vectors, and the difference score between the phase vectors. Both were nearly identical to the contour correlations ( $r>.96$ for both experiments), and thus represented the same information. For conceptual simplicity and avoidance of collinearity, the analyses use only the correlation coefficient.
} 
(a) p1t1: Pitch original, Time original

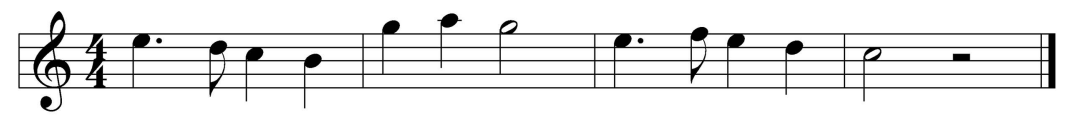

(b) p1t2: Pitch original, Ametric original rhythm

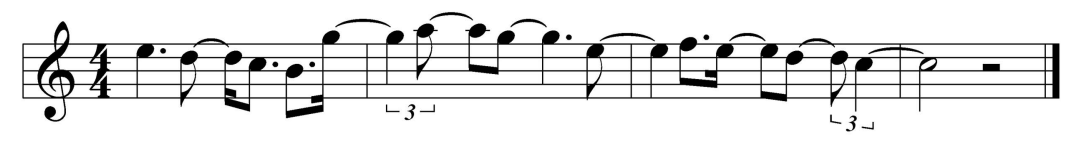

(c) p1t3: Pitch original, Rhythm-violated

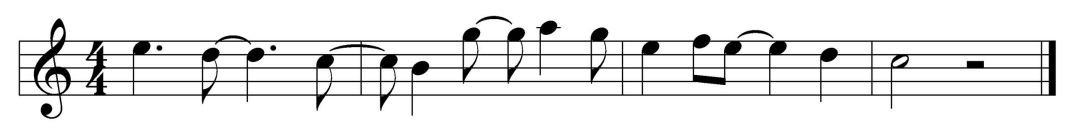

(d) p2t1: Atonal original contour, Time original

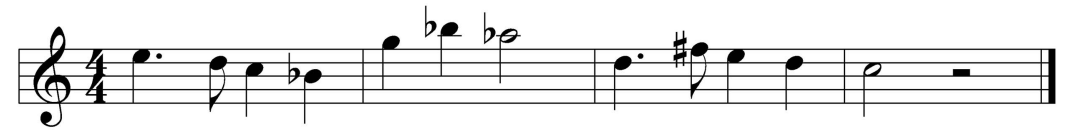

(e) p2t3: Atonal original contour, Rhythm-violated

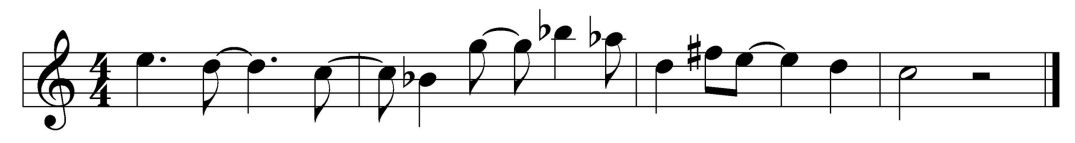

(f) p3t1: Contour-violated, Time original

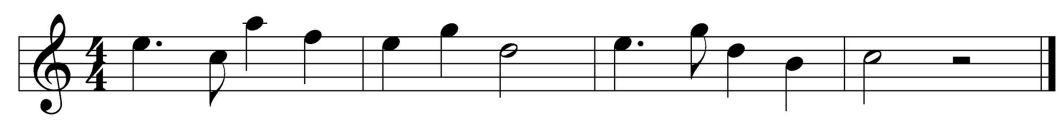

(g) p3t2: Contour-violated, Ametric original rhythm

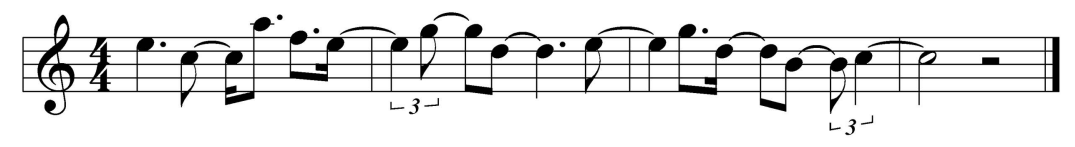

(h) p4t4: Contour-violated-atonal, Rhythm-violated-ametric

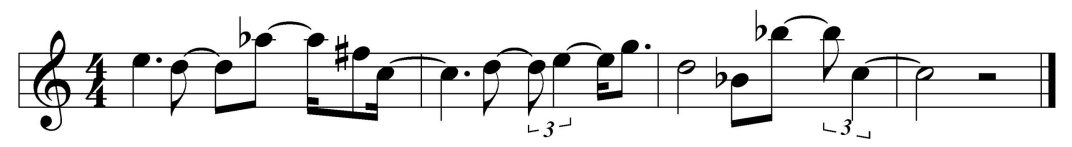

Figure 1. Example melody variants for Experiment 1. Sequences were always transposed within a given trial, such that they would start on different notes, but for ease of comparison are not transposed here.

predictor was the average absolute difference in the correlation with the metric hierarchy (Palmer \& Krumhansl, 1990) between the two melodies. As larger tonal and/or metric difference should result in lower similarity ratings, negative correlations were expected between these predictors and similarity ratings.

The final analysis approach involved exploratory factor analysis using principal components analysis of the $16 \times 16$ matrix of perceived similarity ratings averaged across participant (following Monahan \& Carterette, 1985). These techniques allow extraction of the underlying factors that explain the similarity ratings while making no assumptions about the nature of the stimuli or experimental manipulations (Kruskal \& Wish, 1978). The extracted factors are then inspected for the extent to which they resemble the manipulated differences between the melodies. 


\section{Results}

Preliminary checks. To determine whether participants were able to notice changes in melodic similarity (that is, that the task was not too difficult), the average ratings for the 16 exact match conditions (e.g., p2t3-p2t3) were compared with the average rating from all 240 nonmatch conditions. Regardless of participants' use of surface and structure information in pitch and time, they should rate exact match conditions as more similar than nonmatches. Reassuringly, the average similarity rating for the match conditions was $5.71(S D=.46)$, compared with $4.30(S D=.81)$ for the nonmatches, demonstrating that participants were indeed sensitive to alterations to the melodies, $t(33)=16.6, p<.001$ (all $t$ tests are two-tailed paired samples). Note that some nonmatches were relatively similar, such as p1t1-p1t2, so an average rating of 4.30 for all nonmatches is not unreasonable - by comparison, the average p1t1-p4t4 rating was 2.72. Figure 2 shows a gray-scale plot of the $16 \times 16$ similarity matrix, averaged across participant. This figure reflects the fact that perceived similarity between melody pairs is high along the ascending diagonal (match conditions), and decreases with surface and structural differences in both dimensions. Also note the uniformly low ratings of the descending diagonal (conditions in which both the pitch and time levels were maximally different).

Order effects. Figure 2 is also useful for assessing the possibility of order effects - that the similarity rating between two variant types (e.g., p1t2 and p4t3) varies based on which type occurred first. Cells below the ascending diagonal (lower triangle) represent conditions in which the variant with fewer changes to the original melody (e.g., p1t2) is heard first, whereas above the diagonal (upper triangle) shows the variant with more changes (e.g., p4t3) first. It is possible that hearing a more typical melody followed by a less typical one would result in lower similarity ratings than the other direction (Bartlett \& Dowling, 1988), because "good patterns have few alternatives" (Garner, 1970, p. 39). Indeed, in the present data, the average similarity rating of the lower triangle conditions was significantly lower than those in the

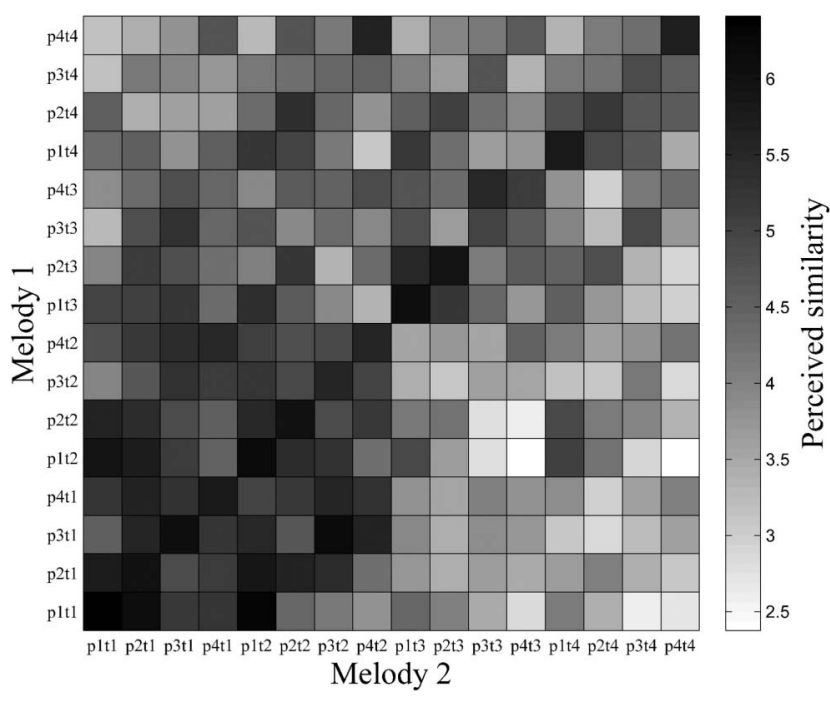

Figure 2. Plot of Experiment 1 similarity ratings. The ascending diagonal represents match conditions (e.g., p3t2-p3t2), and accordingly has the highest similarity ratings. upper triangle, $t(33)=4.1, p<.001$, although the mean difference between triangles was only 0.2 (lower, $M=4.2, S D=.61$; upper, $M=4.4, S D=.57$ ).

Given this overall mean difference, further examination tested whether the ratings in the lower and upper triangles followed the same pattern - that is, if the contribution of pitch and time manipulations changed as a function of variant order. The selected approach was to compare the consistency among participants (i.e., random variation) to that between the lower and upper triangles (variant order). Put differently, was the variation in ratings based on variant order (upper or lower triangle) comparable with what one would predict based on random variation between participants? Each participant experienced one of two possible variant order combinations, as participants did not hear all 256 variant order combinations (cf. last paragraph of Stimuli in the Method section). Therefore, rating consistency had to be calculated separately for the two variant order combinations, grouping together participants who experienced the same variant orders. To examine the random variation between participants, each group was split into two subgroups (random assignment), whose ratings were averaged separately and correlated (using only the 120 nondiagonal cells). Participant subgroups intercorrelated at $r(118)=.60$ (for variant order Group 1) and .64 (for variant order Group 2), both $p s<.001$. This measure of the random between-participants variation was comparable with the correlation of the lower and upper triangles (averaging across participant), $r(118)=.59, p<.001$. In other words, ratings varied as much between participants (of a given group) as they did across the overall lower-upper triangle, suggesting that the order effects did not change qualitatively the similarity ratings.

Expertise analysis. Testing whether musically trained participants emphasized structural information more than untrained listeners began with calculating the zero-order correlations between each participant's ratings and the theoretical predictors. This gave 34 coefficients for each variable (contour, rhythm, tonality, and meter), indicating how influential each variable was for each participant. The second step was to correlate these values with years of musical training, which revealed how the contribution of each variable changed as a function of expertise. The strongest of these correlations was a trend toward greater sensitivity to contour for musically trained participants, $r(32)=.31, p=.077$, but not rhythm, $r(32)=.01, p=$ .958. There was no significant association between expertise and use of tonality, $r(32)=-.26, p=.130$, nor between expertise and meter, $r(32)=-.20, p=.267$. In other words, musically trained participants trended toward better use of surface information in their ratings of perceived melodic similarity, but not for any other variable (time surface, pitch structure, time structure).

Categorical ANOVA analysis. Testing for categorical effects of surface and structure of pitch and time on similarity ratings used a $2 \times 2 \times 2 \times 2$ univariate ANOVA of contour, tonality, rhythm, and meter (for all variables, the levels were same, or different). In this analysis there were main effects of contour, $F(1,240)=83.7$, $p<.001, \eta^{2}=.14$, and rhythm, $F(1,240)=190.3, p<.001$, $\eta^{2}=.31$, but not tonality, $F(1,240)=2.2, p=.134, \eta^{2}<.01$, or meter, $F(1,240)=1.2, p=.273, \eta^{2}<.01 .^{4}$ That is, both

\footnotetext{
${ }^{4}$ All $\eta^{2}$ values are full (not partial) eta-squared, using the corrected total Type III sum of squares.
} 
Table 3

Interaction Between Rhythm and Meter

\begin{tabular}{lcccc}
\hline & $\begin{array}{c}\text { Experiment 1: } \\
\text { Meter same }\end{array}$ & $\begin{array}{c}\text { Experiment 1: } \\
\text { Meter different }\end{array}$ & $\begin{array}{c}\text { Experiment 2: } \\
\text { Meter same }\end{array}$ & $\begin{array}{c}\text { Experiment 2: } \\
\text { Meter different }\end{array}$ \\
\hline Rhythm same & $5.14(.08)$ & $5.21(.11)$ & $4.38(.08)$ & $4.63(.12)$ \\
Rhythm different & $\underline{4.19(.08)}$ & $\underline{3.94(.06)}$ & $3.93(.08)$ & $3.86(.07)$ \\
\hline
\end{tabular}

Note. Underlined values indicate the condition in which metrical similarity affected perceived similarity (see text). Standard error of the mean values are in parentheses.

surface variables were significant, but neither structural variable was significant. Only one interaction-between rhythm and meter, $F(1,240)=3.9, p=.050, \eta^{2}<.01-$ met the threshold of significance, reflecting the pattern that preserving meter only raised perceived similarity when the rhythm was different between melodies (meter had no effect when rhythm stayed constant; see Table 3). Contour and tonality approached a significant interaction, $F(1,240)=3.4, p=.066, \eta^{2}<.01$, and followed the opposite pattern of surface and structure, that is, tonality marginally increased similarity only when the contour was the same, and was completely ineffective when contour changed. Table 4 shows this (nonsignificant) pattern. No other interactions were significant (all $F \mathrm{~s}<1)$.

Figure 3 shows the similarity ratings for all pitch-level combinations averaged across participant and across time levels (i.e., all combinations of the four pitch manipulation levels); Figure 4 provides the complement for time. These figures show the same relative patterns of perceived similarity, such that the values along the ascending diagonal (matching levels) are most similar, with decreasing similarity toward the opposite corners.

A potential concern from Figure 4 is that participants may have been unable to differentiate between the first two levels of temporal manipulation ( $\mathrm{t} 1=$ original; $\mathrm{t} 2=$ ametric original rhythm). Indeed, when presented with irregular timing intervals, listeners tend to regularize them to a standard metrical framework (Motz, Erickson, \& Hetrick, 2013; Repp, London, \& Keller, 2011). Figure 2 shows high similarity between p1t1-p1t2 (and the reverse order), and Figure 4 shows high similarity between $\mathrm{t} 1$ and $\mathrm{t} 2$ variants. However, the confidence intervals (CIs) associated with Figure 4 show that participants gave significantly higher similarity ratings to t1-t1 pairs $(M=5.50,95 \%$ CI $[5.32,5.69])$ than $\mathrm{t} 1-\mathrm{t} 2(M=$ $5.19,95 \%$ CI [4.99 5.39]) and t2-t1 pairs $(M=5.12,95 \%$ CI [ 4.93 5.30]).

Following the main effect of order observed in the overall data, the difference between mean similarity ratings of the lower triangle $(4.1, S D=.65)$ and upper triangle $(4.3, S D=.58)$ of Figure 3 (pitch variant levels) was significant, $t(33)=3.3, p=.002$. In addition, as before, the pattern of similarity across levels was alike:
The upper and lower triangles of Figure 3 correlate at $r(4)=.79$. For time, the similarity ratings of the cells in the lower triangle $(M=4.0$, $S D=.69)$ of Figure 4 were also lower than those in the upper triangle $(M=4.4, S D=.66), t(33)=3.4, p=.002$. The pattern of ratings correlated highly across order, $r(4)=.88$. These analyses reaffirm that although the range of similarity ratings varied across order (i.e., there was a main effect of order), the pattern across pitch and time levels remained the same.

Regression analysis. The second analysis approach of linear regression equation predicted the similarity ratings using measures of contour, rhythm, tonality, and meter (see Data Analysis section of Method for details). As signed predictors of tonality and meter were not related to ratings ( $r=-.05$ and -.06 , respectively), only the absolute difference values were included in the regression. Table 5 shows the final equation, which explained $60 \%$ of the variance using the rhythm, contour, and metricality predictors (in order of contribution strength), with the expected coefficient sign. Tonality was not a significant predictor of perceived similarity, despite a significant zero-order correlation, $r=-.18, p=.005$. The number of shared pitch classes between melodies also did not explain any of the variance in ratings (by design). Four multiplicative interaction predictors were also tested: contour and rhythm (pitch and time surface), tonality and meter (pitch and time structure), contour and tonality (pitch surface and structure), as well as rhythm and meter (time surface and structure). None contributed any unique variance beyond the existing predictors.

Factor analysis. Four factors (all eigenvalues above 1) explained $87 \%$ of the variance in the ratings (see Table 6 for factor scores). To interpret the identity of factors, the factor scores were compared with the predictors from the regression equation (contour, tonality, rhythm, and meter), following Eerola et al. (2001). This required converting the factor scores into distances by calculating pairwise differences between all possible combinations of the 16 variants (p1t1-p1t2, p1t1-p1t3, .., p4t4-p4t4), yielding a 256-element vector for each factor. The absolute value of these distances (higher numbers representing greater distance in the factor space) was then correlated with the regression predictors, giving the values shown in Table 7 . The highest correlations (in

Table 4

Interaction Between Contour and Tonality

\begin{tabular}{lcccc}
\hline & $\begin{array}{c}\text { Experiment 1: } \\
\text { Tonality same }\end{array}$ & $\begin{array}{c}\text { Experiment 1: } \\
\text { Tonality different }\end{array}$ & $\begin{array}{c}\text { Experiment 2: } \\
\text { Tonality same }\end{array}$ & $\begin{array}{c}\text { Experiment 2: } \\
\text { Tonality different }\end{array}$ \\
\hline Contour same & $5.12(.08)$ & $4.85(.11)$ & $\underline{4.78(.08)}$ & $\underline{4.31(.12)}$ \\
Contour different & $4.24(.08)$ & $4.27(.06)$ & $3.91(.08)$ & $3.80(.07)$ \\
\hline
\end{tabular}

Note. Underlined values indicate the condition in which tonal similarity affected perceived similarity (see text). Standard error of the mean values are in parentheses. 


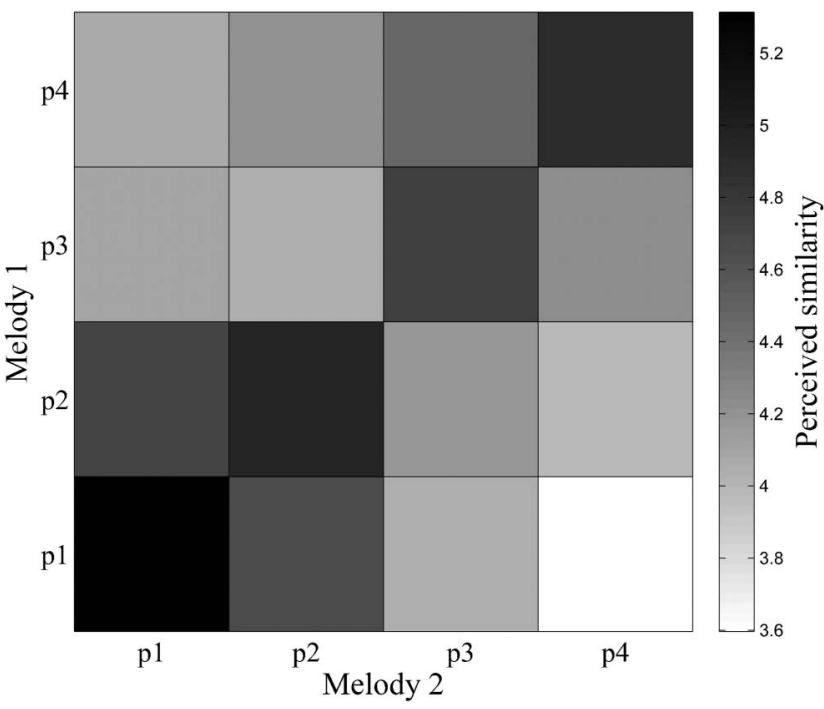

Figure 3. Perceived similarity of all pitch manipulation levels in Experiment 1 , averaged across time manipulation levels. Note the resulting change in color scale from Figure 2.

bold) denote the predictor with which each factor correlated best, ${ }^{5}$ which turned out to be rhythm, contour, meter, and tonality, respectively.

Figures 5 and 6 provide a visualization of the four factor scores as two-dimensional similarity maps. The coordinates from the temporal factors of rhythm and meter (Factors 1 and 3) are depicted in Figure 5; Figure 6 shows the coordinates from the pitch factors of contour and tonality (Factors 2 and 4). Overall, the factor analysis demonstrates that the four independent extracted factors correspond to the surface and structural stimulus manipulations, and explain the perceived similarity ratings remarkably well.

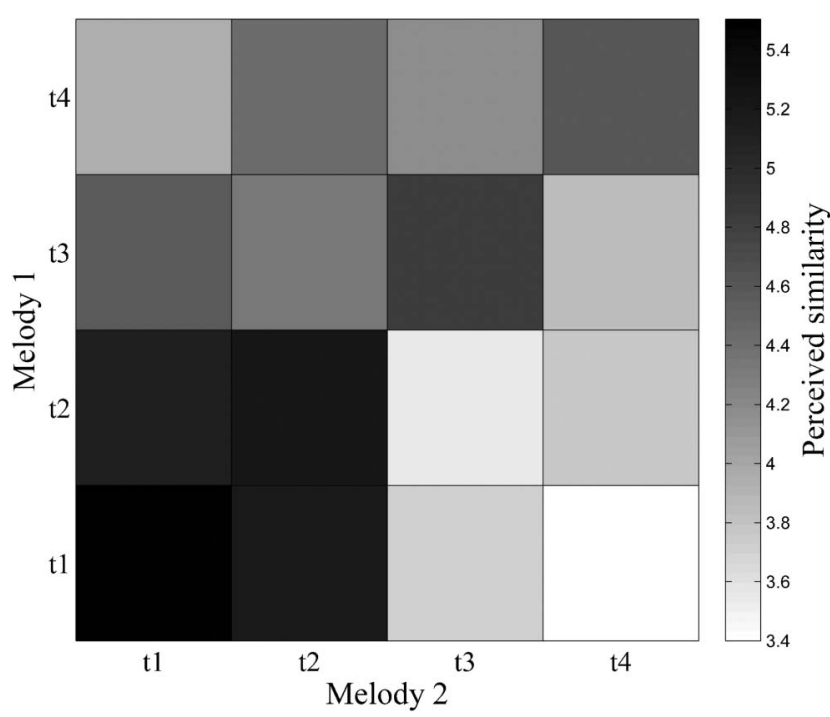

Figure 4. Perceived similarity of all time manipulation levels in Experiment 1 , averaged across pitch manipulation levels.

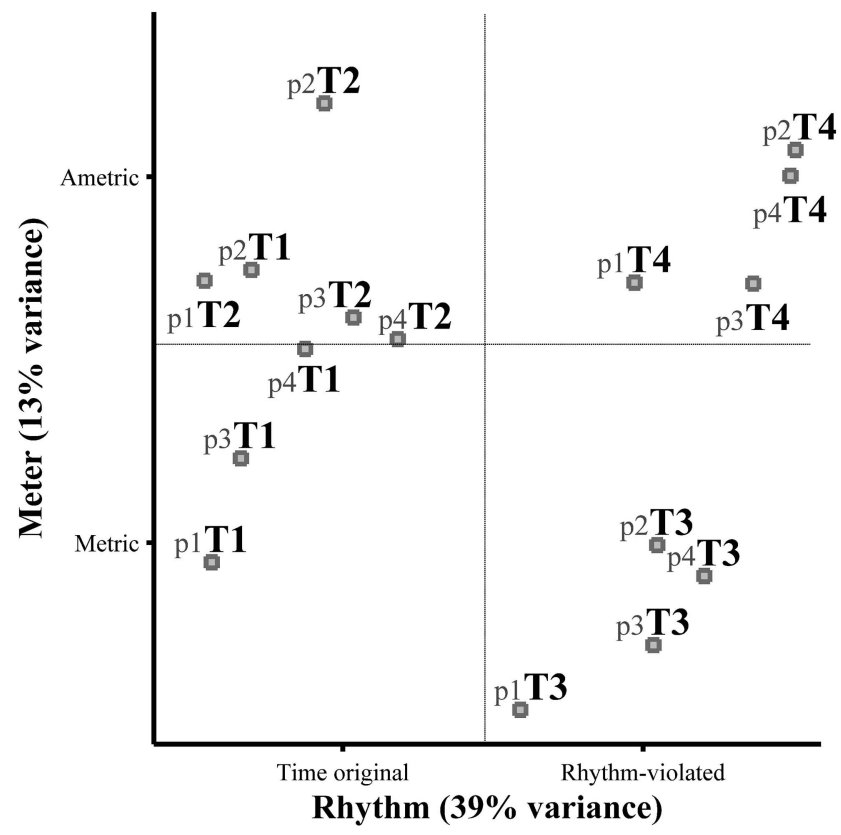

Figure 5. Factors 1 and 3 (interpreted as rhythm and meter) of the factor analysis solution of perceived similarity ratings of all compared variants, for Experiment 1. For clarity, data labels emphasize time levels, and internal axes crossing at the origin are added.

\section{Discussion}

The factorial manipulations of melodic surface and structure in both pitch and time (contour, rhythm, tonality, and meter) allowed investigation of their respective and combined roles in perceived similarity. There were four results of particular importance. First, although both surface and structural information contributed to ratings, rhythm and contour (surface information) were the primary determinants of perceived similarity. Second, order effects were slight and theoretically inconsequential. Third, despite the central role of pitch in Western music, temporal factors were the stronger predictors of melodic similarity in all analyses. Fourth, the predictors functioned essentially independently.

The predominance of surface information is consistent with findings in the perception of unfamiliar music (Eerola et al., 2001; Halpern, 1984; Lamont \& Dibben, 2001; McAdams et al., 2004). It is likely that with increased exposure to the same melodies, structural information would become a stronger contributor to perceived similarity, as previous authors have demonstrated (Pollard-Gott, 1983; Serafine et al., 1989). However, it seems unlikely that increased musical training would play a role, as expertise was not associated with greater sensitivity to either form of structure. If anything, musicians were slightly better at noticing contour (surface) changes, but not at the expense of structural

\footnotetext{
${ }^{5}$ The negative sign of the coefficients with rhythm and contour in Table 7 emerges because larger factor scores (i.e., greater distance) correlate negatively with these predictors, in which larger values denote greater similarity (smaller distance). Likewise, the values are positive in Columns 3 and 4 , as higher numbers in meter and tonality predictors indicated greater distance. In all cases, the sign is consistent with the theoretical prediction. The same applies to Table 10 (Experiment 2).
} 
Table 5

Experiment 1 Regression Equation

\begin{tabular}{lccccrr}
\hline & Standardized Beta & $t$ & $p$ & Zero-order correlation & $s r^{2}$ & Tolerance \\
\hline Intercept & & 37.125 & .000 & & & \\
Rhythm & .570 & 12.934 & .000 & .613 & .268 & .823 \\
Contour & .461 & 11.522 & .000 & .462 & .212 & 1.000 \\
Metricality & -.100 & -2.266 & .024 & -.339 & .008 & .823 \\
Total $r^{2}=.597$ & & & & & & \\
\hline
\end{tabular}

information. This finding is more consistent with a generalized increase in ability to process melodic information as a result of greater skill in musical tasks.

Asymmetries in similarity ratings can occur when one stimulus is less structured than the other (Garner, 1974). Bartlett and Dowling (1988) observed this effect in a musical context when comparing tonal (structured) and atonal (unstructured) melodies. The current experiment shows a consistent pattern, in that similarity ratings were lower when the less altered variant occurred first, but it seems not to have affected the contributions of surface and structure in pitch and time, as the patterns were alike on either side of the diagonals of Figures 2 through 4 . Thus, there was an overall magnitude change in ratings based on order, which did not alter how listeners evaluated similarity in theoretical terms. That is, order effects occurred, but there is no evidence that they influenced the main theoretical question of interest, which is how listeners used contour, tonality, rhythm, and meter in rating melodic similarity.

The extent of the predominance of temporal variables is striking, given the fundamental importance of contour and tonality in music perception (Dowling, 1978; Schmuckler, 2004). As discussed in the introduction, most work has found that temporal features dominate melodic similarity ratings of unfamiliar melodies, but there are reports of pitch being more important in similarity ratings and recognition (e.g., Carterette, Kohl, \& Pitt, 1986; Hébert \& Peretz, 1997; Jones et al., 1987).

Table 6

Factor Scores from Principal Components Analysis of Experiment 1 Ratings (Plotted in Figures 5 and 6)

\begin{tabular}{lrrrr}
\hline Variant & Rhythm & Contour & Meter & Tonality \\
\hline p1t1 & -1.24 & -0.65 & -1.01 & -0.30 \\
p2t1 & -1.06 & -0.04 & 0.59 & 0.04 \\
p3t1 & -1.11 & 1.30 & -0.44 & 0.07 \\
p4t1 & -0.82 & 0.94 & 0.16 & 0.58 \\
p1t2 & -1.27 & -1.23 & 0.53 & -0.50 \\
p2t2 & -0.73 & -0.76 & 1.50 & 0.51 \\
p3t2 & -0.60 & 1.06 & 0.33 & -0.83 \\
p4t2 & -0.40 & 0.87 & 0.21 & 0.83 \\
p1t3 & 0.15 & -1.17 & -1.81 & 0.14 \\
p2t3 & 0.76 & -1.10 & -0.91 & 2.25 \\
p3t3 & 0.75 & 0.61 & -1.46 & -1.57 \\
p4t3 & 0.98 & 0.86 & -1.08 & 0.49 \\
p1t4 & 0.66 & -1.37 & 0.52 & -1.13 \\
p2t4 & 1.39 & -0.89 & 1.25 & 0.03 \\
p3t4 & 1.20 & 0.33 & 0.52 & -1.61 \\
p4t4 & 1.36 & 1.25 & 1.10 & 1.02 \\
\hline
\end{tabular}

Note. Columns are sorted in order of variance accounted for; labels are post hoc interpretations. Rows are sorted first by time level.
Pitch and time were independent in this experiment. Of the 11 interaction terms in the ANOVA (six 2-way, four 3-way, one 4-way), only the two within-dimension terms even approached significance (rhythm-meter and contour-tonality). Additionally, none of the regression interaction terms were significant. By itself, the existence of four factors in the factor analysis does not provide evidence of independence, because the technique is specifically designed to extract independent predictors. Nonetheless, the fact that $87 \%$ of the total variance was explained with these independent factors that mapped well onto the manipulations reinforces the independence found in the other analyses. These mappings were not perfect, as occasional points were counterintuitive (p1t2 is on the wrong side of the $x$-axis in Figure 5, as are p1t3 and p3t in Figure 6). These exceptions represent conflicts with the accordingly weaker (i.e., structural) dimensions.

Variations in observed independence or interaction of pitch and time may stem from unequal discriminability (Garner \& Felfoldy, 1970), or from one dimension being more salient than another (Prince, Thompson, et al., 2009). Indeed, sufficiently imbalanced dimensional salience (e.g., via changes in stimulus structure, or task) can obscure otherwise observable pitch-time interactions (Prince, 2011; Prince, Schmuckler, \& Thompson, 2009). Perhaps in this experiment, temporal variables were sufficiently stronger than pitch variables so as to suppress any observable interaction between dimensions. In particular, the fact that the melodies all had the same tempo means that both relative and absolute timing information was available for use in evaluating similarity. For example, a p3t2-p4t2 comparison not only had the same sequence of duration ratios but also had exactly the same durations. Using a constant tempo was a deliberate choice in Experiment 1, so that the total elapsed duration of both melodies in a pair remained constant. In comparison, transposing the melodies to different keys preserved only the relative pitch patterns, not the exact pitch classes. Therefore, the temporal dimension was in a sense more reliable,

Table 7

Correlations Between Distances Calculated Using Factor Scores and Regression Predictors for Experiment 1

\begin{tabular}{|c|c|c|c|c|}
\hline & Rhythm & Contour & Meter & Tonality \\
\hline Factor 1 (39\% variance) & $-0.78^{* * *}$ & -0.04 & $0.32^{* * * *}$ & 0.10 \\
\hline Factor 2 (28\% variance) & -0.06 & $-0.74^{* * *}$ & 0.04 & 0.07 \\
\hline Factor 3 (13\% variance) & $-0.37^{* * * *}$ & -0.02 & $0.41^{\text {******* }}$ & $0.19^{* * *}$ \\
\hline Factor 4 (7\% variance) & $-0.18^{* *}$ & $-0.23^{* * * *}$ & 0.05 & $0.42^{* * * *}$ \\
\hline
\end{tabular}

Note. Columns are ordered by the percent variance accounted for by the assigned factor, as determined by which predictor had the highest correlation with each factor (bolded diagonal values)

${ }^{* * *} p<.01 .{ }^{* * * *} p<.001$. 


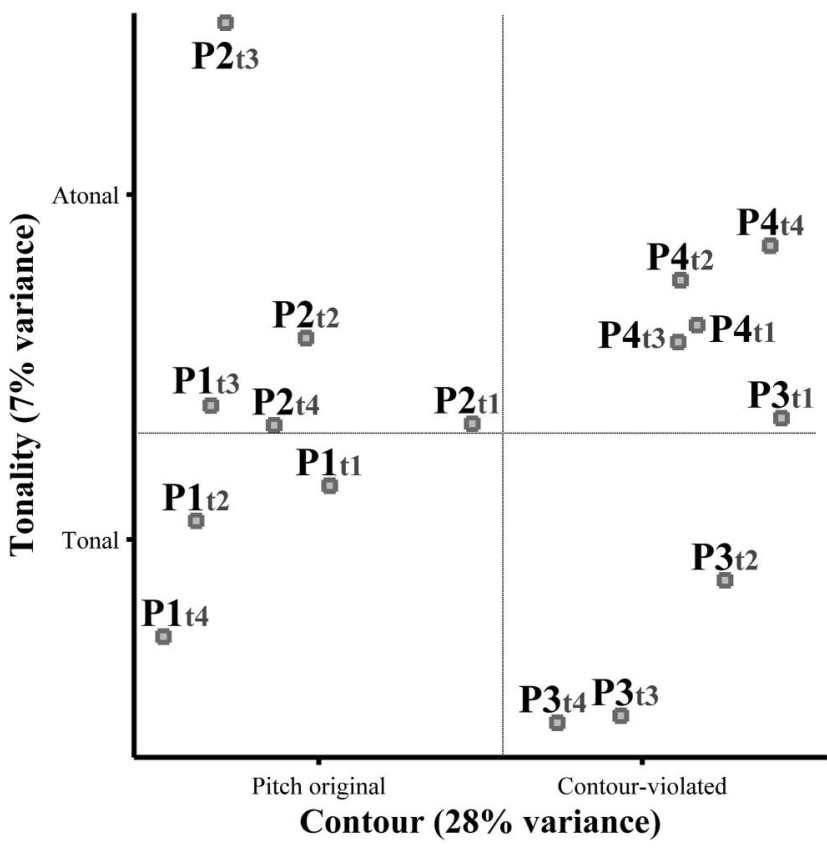

Figure 6. Factors 2 and 4 (interpreted as contour and tonality) of the factor analysis solution of perceived similarity ratings of all compared variants, for Experiment 1. For clarity, data labels emphasize pitch levels, and internal axes crossing at the origin are added.

providing more stable cognitive reference points for listeners to use in rating melodic similarity.

Transposition provided a further handicap to the pitch dimension of these melodies, because after hearing the first melody in one key, listeners then had to reorient to a new key when the second melody started. Even if both melodies in the pair are tonal, the second melody will sound atonal until the listener adjusts to the new key, decreasing the perceived similarity accordingly; in most cases, there would also be carryover effects onto the first melody of the next trial. Thus, the effects of transposition may have decreased the informative value of pitch, causing a relative increase in salience of time. In turn, a sufficiently high imbalance in dimensional salience may have reduced the chance of observing pitch-time interactions.

Experiment 2 tested the effects of tempo change and transposition on perceived melodic similarity in order to address these issues and further explore how listeners use pitch and time in this context.

\section{Experiment 2}

There were two alterations to the Experiment 1 stimulus melodies in Experiment 2. First, the two melodies within a trial were played at different speeds. Second, a chord cadence preceded each melody, which established both the upcoming key and tempo before the melody itself began. One result of these changes is that listeners had only relative timing and relative pitch cues to evaluate similarity, instead of also preserving absolute timing information. Another important implication is that by establishing both the new key and tempo before the melody started, structurepreserving variants would not appear as unstructured, having ad- justed to the new tonal center and metrical framework before the melody started. There were no other changes to the stimuli or the experimental design.

\section{Method}

Participants. A new set of 34 participants were recruited for Experiment 2, with an average age of $25.9(S D=8.5)$ and 2.8 years of musical training $(S D=4.1)$. Participants were again recruited from the Murdoch University community, and provided with modest financial compensation or course credit.

Stimuli. As noted previously, stimuli were the same melodies from Experiment 1, but with a chord cadence prefix and at one of two different tempi. The faster melodies were from Experiment 1; slower versions were added (two thirds the speed of the fast melodies). The durations of each note in the slower melodies were either $250 \mathrm{~ms}$ (eighth note), $500 \mathrm{~ms}$ (quarter note), $750 \mathrm{~ms}$ (dotted eighth note), or 1,000 ms (half note). The chord cadence (I-V-I cadence, transposed to the appropriate key) was always tonal, to prevent a confound with the tonality manipulation of the melody (see Figure 7 for an example). Tempo order (slow-fast or fastslow) was counterbalanced throughout the experiment and across participant. Minor mode melodies had a harmonic minor I-V-I cadence.

Procedure. The Experiment 2 procedure was the same as that of Experiment 1. Participants were instructed to rate the similarity of the melodies and disregard the chord cadence prefix. They waited an average of $1.0 \mathrm{~s}$ between melodies $(S D=.36$, median $=$ .97). Due to the longer stimuli and slower melodies, average completion time increased to $45 \mathrm{~min}$.

Data analysis. The data analysis approaches were the same as in Experiment 1.

\section{Results}

Preliminary checks. The average rating for the 16 match conditions was $5.04(S D=.64)$, compared with $3.97(S D=.75)$ for the 240 nonmatch conditions, indicating that participants were able to complete the task successfully, $t(33)=10.1, p<.001$. Figure 8 is a greyscale plot of the $16 \times 16$ similarity matrix, showing, as in Experiment 1, that the diagonal (match) conditions received the highest similarity ratings, which decreased away from the diagonal. The axes have been reordered from Experiment 1 in accordance with which dimension was more influential (time for Experiment 1; pitch for Experiment 2). This reordering does not

(a) p1t1: Pitch original, Time original

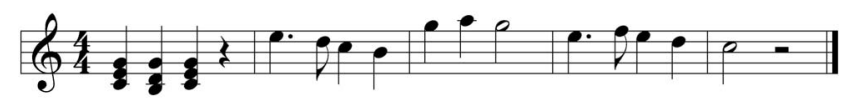

(b) p2t3: Atonal original contour, Rhythm-violated

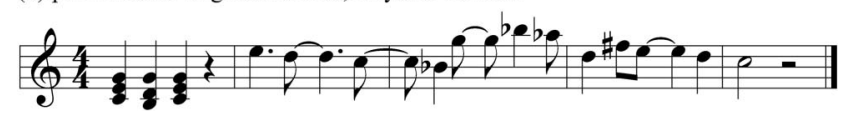

Figure 7. Example variants used in Experiment 2. The stimuli were the same as Experiment 1 (see Figure 1), except for the added chord cadence prefix and variable tempo (across melody). 


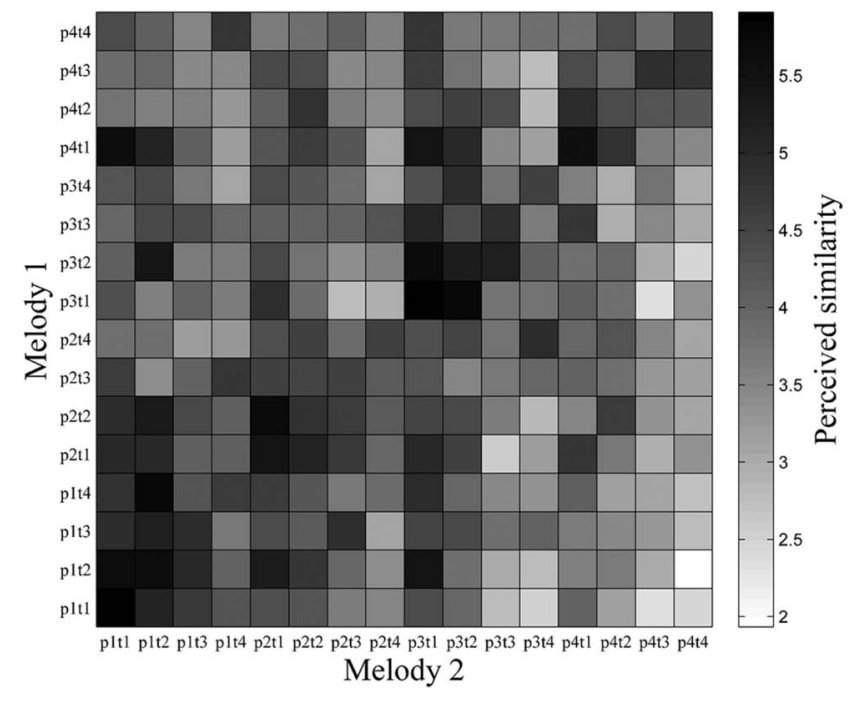

Figure 8. Plot of Experiment 2 similarity ratings. The ascending diagonal represents match conditions, which have the highest similarity ratings. The axes have been reordered from the Experiment 1 data (see Figure 2) in accordance with the relative explanatory value of dimensions in similarity ratings.

change any analyses, but is intended to display the decreasing similarity from the diagonal (match conditions) more clearly.

Order effects. The ratings of the lower-triangle (more stable variant first) conditions $(M=3.8, S D=.79)$ were significantly lower than the upper-triangle conditions $(M=4.2, S D=.76)$, $t(33)=5.0, p<.001$, indicating the presence of an order effect. The correlation between upper and lower triangles $(r=.27)$ was lower than the subgroup intercorrelations $(r=.44 ; .56)$, when calculated as described in Experiment 1.

Expertise analysis. The same analysis technique as in Experiment 1 revealed that musical training enhanced the use of contour, $r(32)=.39, p=.021$ (two-tailed), but not rhythm, $r(32)=-.22$, $p=.202$. There was no significant association between expertise and use of tonality, $r(32)=-.07, p=.679$, nor between expertise and meter, $r(32)=-.251, p=.152$. Thus, again, musically trained participants were better able to make use of contour information in perceived similarity ratings, but no other differences emerged across expertise.

Categorical ANOVA analysis. The $2 \times 2 \times 2 \times 2$ univariate ANOVA testing the effects of pitch and time manipulations on similarity ratings revealed main effects of contour, $F(1,240)=$ $60.2, p<.001, \eta^{2}=.12$, rhythm, $F(1,240)=47.1, p<.001$, $\eta^{2}=.09$, and tonality, $F(1,240)=11.0, p=.001, \eta^{2}=.02$, but not meter, $F(1,240)=1.0, p=.309, \eta^{2}<.01$. Only the interaction between contour and tonality was significant, $F(1$, $240)=4.0, p=.046, \eta^{2}=.01$; the rhythm-meter interaction approached, but did not reach, the threshold, $F(1,240)=3.2, p=$ $.075, \eta^{2}=.01$. No other interactions were significant. The similarity ratings associated with the main effect of pitch manipulations (averaged across time levels) are depicted in Figure 9; Figure 10 has the same for time.

Listeners differentiated more between pitch variants when the less-altered variant was heard first - that is, the lower triangle of Figure 9 has overall lower similarity ratings $(M=4.1, S D=.65)$

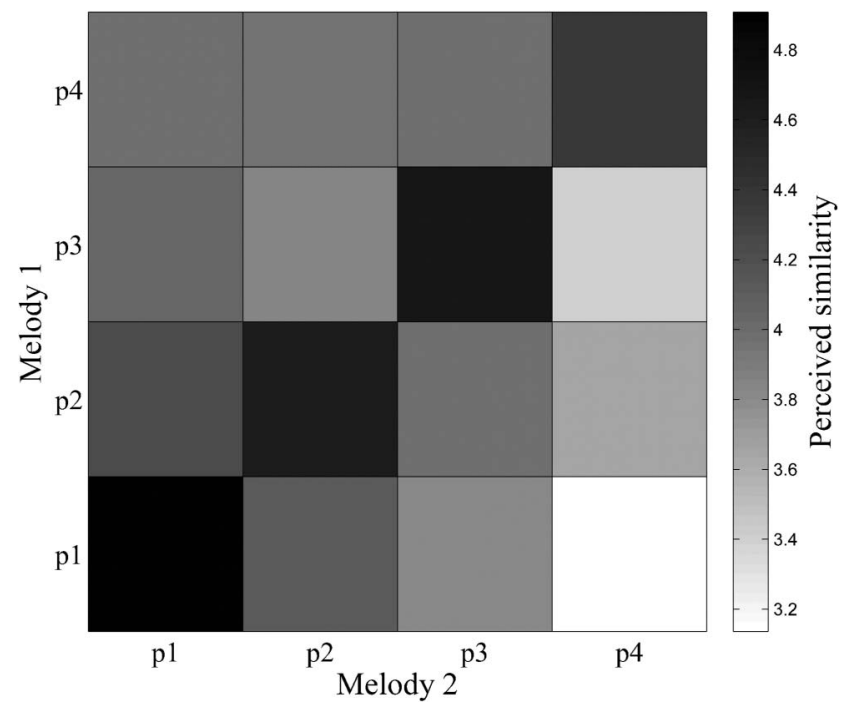

Figure 9. Perceived similarity of all pitch manipulation levels in Experiment 2, averaged across time manipulation levels.

than the upper triangle $(M=4.3, S D=.58), t(33)=4.5, p<.001$. Similarly for time, the lower triangle of Figure 10 received significantly lower ratings than the upper triangle $(M$ lower $=3.7$, $S D=.73 ; M$ upper $=4.2, S D=.87), t(33)=5.3, p<.001$. For both pitch and time, the upper and lower triangles were positively correlated, $r(4)=.34$ and .35 , respectively, showing agreement across order (albeit less than in Experiment 1).

Regression analysis. Regressing objective similarity predictors on the 256 similarity ratings averaged across participant gave significant effects of contour, rhythm, and tonality (absolute, not signed), but not meter; see Table 8 for the equation details. Additionally, the number of shared pitch classes between melody pairs was not a significant predictor of similarity ratings. In total,

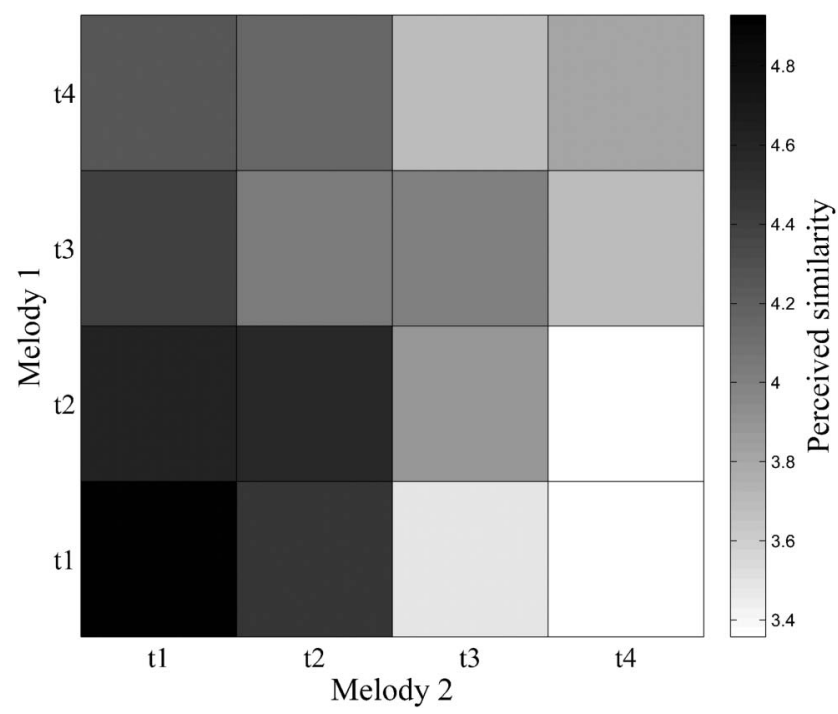

Figure 10. Perceived similarity of all time manipulation levels in Experiment 2, averaged across pitch manipulation levels. 
Table 8

Experiment 2 Regression Equation

\begin{tabular}{lcccccr}
\hline & $\begin{array}{c}\text { Standardized } \\
\text { Beta }\end{array}$ & $t$ & $p$ & $\begin{array}{c}\text { Zero-order } \\
\text { correlation }\end{array}$ & $s r^{2}$ & Tolerance \\
\hline Intercept & & 34.935 & .000 & & & \\
Contour & .413 & 7.791 & .000 & .450 & 0.161 & .944 \\
Rhythm & .320 & 6.214 & .000 & .329 & 0.102 & .997 \\
Tonality & -.155 & -2.914 & .004 & -.268 & 0.023 & .942 \\
Total $r^{2}=.350$ & & & & & & \\
\hline
\end{tabular}

the equation accounted for $35 \%$ of the variance, less than the $60 \%$ of Experiment 1. No interaction terms accounted for additional variance. Note that pitch factors now had a much stronger relative contribution to ratings than the previous experiment.

Factor analysis. Principal components factor analysis of the $16 \times 16$ similarity matrix yielded five factors with eigenvalues greater than 1 (see Table 9 for factor scores). Only the first three factors were interpretable, as contour, tonality, and rhythm, in order of variance accounted for (see Table 10 for correlations between factor scores and regression predictors). Together, these factors account for $61 \%$ of the variance (using the full fivedimensional solution accounts for $77 \%$ ). Figure 11 graphs the variants in a two-dimensional similarity space based on pitch (Factors 1 and 2), whereas Figure 12 depicts rhythm (Factor 3) along with the unexplained Factor 4.

\section{Discussion}

Experiment 2 investigated the roles of surface and structural information in pitch and time on similarity ratings when sequences in a given trial were transposed in tempo as well as key, both of which were prepared by chord cadence prefixes. Thus, only relative information (not absolute) was available in both pitch and time, providing a more conceptually equal basis for comparison between dimensions. This alteration to the task made it longer and

Table 9

Factor Scores From Principal Components Analysis of Experiment 2 Ratings (Plotted in Figures 11 and 12)

\begin{tabular}{lrrrrr}
\hline Variant & Contour & Tonality & Rhythm & Factor 4 & Factor 5 \\
\hline p1t1 & -1.41 & -0.09 & 0.38 & -1.55 & -0.35 \\
p1t2 & -1.83 & -0.20 & -0.38 & -0.12 & 0.00 \\
p1t3 & -0.63 & -0.33 & 0.60 & 0.01 & -2.19 \\
p1t4 & -0.81 & -0.21 & 0.82 & -0.69 & 0.71 \\
p2t1 & -0.95 & 0.90 & -0.90 & 1.07 & 0.54 \\
p2t2 & -1.01 & 0.96 & -0.23 & 1.53 & 0.63 \\
p2t3 & -0.06 & 1.20 & 1.34 & 0.19 & 0.58 \\
p2t4 & 1.14 & 0.41 & 0.79 & 1.96 & 0.10 \\
p3t1 & 0.40 & -2.09 & -0.83 & -0.28 & 1.20 \\
p3t2 & 0.52 & -1.96 & 0.23 & 0.69 & 0.85 \\
p3t3 & 0.47 & -0.51 & 0.92 & -0.14 & 0.43 \\
p3t4 & 0.65 & -0.74 & 0.58 & 0.51 & -2.30 \\
p4t1 & 0.01 & 0.02 & -2.42 & -0.20 & -0.55 \\
p4t2 & 1.44 & 0.77 & -1.16 & -0.22 & -0.36 \\
p4t3 & 1.19 & 1.12 & -0.65 & -1.18 & 0.02 \\
p4t4 & 0.91 & 0.76 & 0.93 & -1.56 & 0.69 \\
\hline
\end{tabular}

Note. Columns are sorted in order of variance accounted for; labels are post hoc interpretations. Note that this order diverges from Experiment 1, and that rows are accordingly sorted first by pitch levels. harder, resulting in more noise in the data; additionally, order effects were stronger than in Experiment 1. Nevertheless, three findings of interest emerged. First, the balance of predictive value shifted away from temporal variables (rhythm and meter) and toward pitch information (contour and tonality). Second, surface information remained more prominent than structure (and expertise did not modify this balance). Third, despite more equalized effects of pitch and time, no interactions between the dimensions emerged.

Compared with Experiment 1, the data of Experiment 2 exhibited larger standard deviations, lower intersubject correlations, smaller ANOVA effect sizes, and smaller amounts of variance explained in regression and factor analysis techniques. Together, these findings all point toward Experiment 2 being more difficult than Experiment 1 for participants. Accordingly, removing the absolute timing information by introducing a tempo change substantially reduced participants' ability to judge the similarity of the variants, which follows from findings that tempo changes impair memory for melodies (Dowling, Bartlett, Halpern, \& Andrews, 2008; Halpern \& Mullensiefen, 2008; Schellenberg et al., 2014).

Order effects were more observable in Experiment 2, as evidenced by lower similarity ratings for conditions that presented a less-altered melody first (i.e., the lower vs. upper triangles of Figures 8 to 10). The order effects show that participants seem to have had greater trouble differentiating between sequences when the first sequence was a variant that departed more from the original (as in Bartlett \& Dowling, 1988; Watkins, 1985). This pattern is especially notable in Figure 10, which depicts the main effects of comparing each time-level manipulation. In this figure, neither levels $\mathrm{t} 3$ nor $\mathrm{t} 4 \mathrm{receive}$ the highest similarity on their diagonal (matching) conditions - that is, the $\mathrm{t} 3-\mathrm{t} 3$ conditions were

Table 10

Correlations Between Factor Scores (Listed With Percent Variance Accounted For) and Regression Predictors for Experiment 2

\begin{tabular}{lllll}
\hline & Contour & Tonality & Rhythm & Meter \\
\hline Factor 1 (28\% variance) &.$- \mathbf{4 3 8}^{* * *}$ & $.165^{* *}$ & -.074 & .102 \\
Factor 2 (19\% variance) & $-.247^{* * * *}$ & $\mathbf{. 5 0 7}^{* * *}$ & -.016 & .048 \\
Factor 3 (14\% variance) & $-.221^{* * * *}$ & .119 & $-.309^{* * *}$ & $.235^{* * *}$ \\
Factor 4 (9\% variance) & $-.153^{*}$ & $.229^{* * *}$ & -.094 & .119 \\
Factor 5 (7\% variance) & -.029 & .051 & $-.124^{*}$ & .031 \\
\hline
\end{tabular}

Note. Ordering the columns by percent variance accounted for with the assigned factor (as in Table 7) yields a different order of predictors than Experiment 1. Factors 4 and 5 have no obvious matching predictor. ${ }^{*} p<.05$. ** $p<.01$. $^{* * *} p<.001$. 


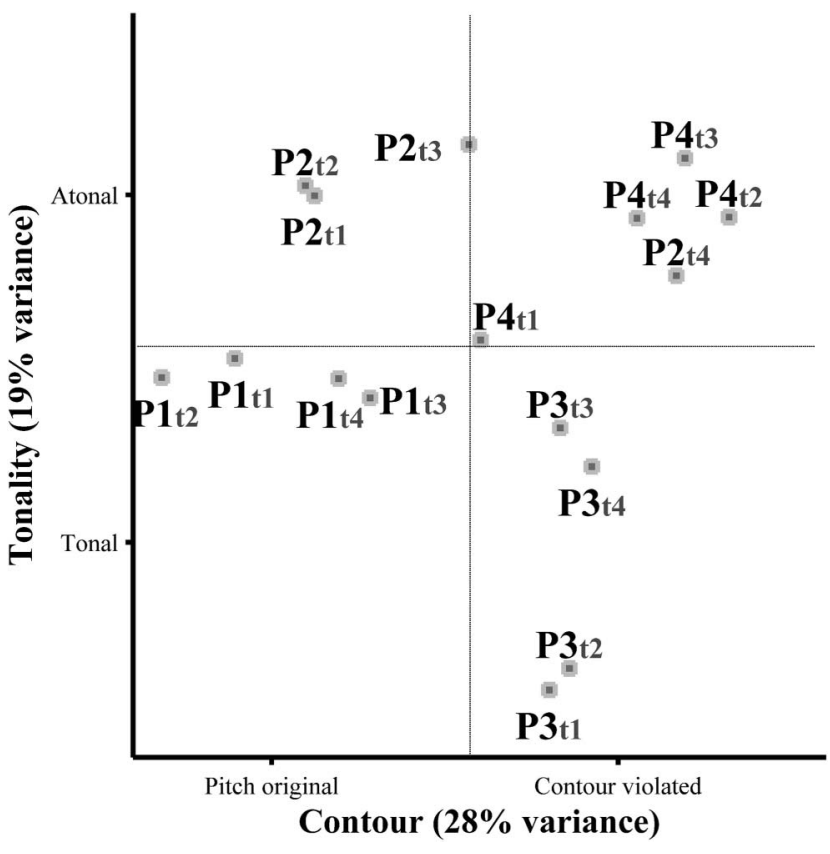

Figure 11. Factors 1 and 2 (interpreted as contour and tonality) of the factor analysis solution of perceived similarity ratings of all compared variants, for Experiment 2. For clarity, data labels emphasize pitch levels, and internal axes crossing at the origin are added.

rated as less similar than $\mathrm{t} 3$-t 1 conditions; likewise, $\mathrm{t} 4-\mathrm{t} 1$ and $\mathrm{t} 4-\mathrm{t} 2$ conditions receive higher similarity ratings than $t 4-t 4$. In all other corresponding figures (Figures 3, 4, and 9), the match conditions were always the most similar.

In Experiment 2, listeners relied more on pitch than time to form their similarity judgments. Specifically, contour was the most influential predictor, whereas rhythm was weaker; tonality was stronger than previously observed, whereas meter no longer contributed to ratings at all. The stronger role of tonality compared with Experiment 1 may represent the removal of the penalty on the dimension of pitch from an unprepared transposition, as transposing a melody to a new key makes recognition more difficult (Dowling \& Fujitani, 1971; Stalinski \& Schellenberg, 2010).

In both experiments, surface information was generally a more powerful predictor of perceived similarity than structural information, although tonality (pitch structure) rivaled that of rhythm (time surface) in Experiment 2. Thus, simply inserting a chord cadence before each melody resulted in a stronger role of structure in Experiment 2, even without repeated presentations of these unfamiliar melodies, which is known to affect the balance between surface and structure (Pollard-Gott, 1983; Serafine et al., 1989). Again, musical expertise aided the participants in recovering contour information when forming their rating, but it did not alter the relative emphasis on surface and structure.

The main goal of Experiment 2 was to see whether putting pitch and time on a more conceptually equal footing altered their relative contribution to perceived similarity, and whether interactions between pitch and time were more apparent when the effect sizes were more equal. From the ANOVA effect sizes and the squared semipartial correlations of the regression, the experiment was successful in altering the weighting of the dimensions toward parity, as pitch and time accounted for a more comparable amount of variance in the data. Nevertheless, pitch-time interactions were no stronger than in the previous experiment, and the factor analysis again retrieved independent factors that corresponded well to the manipulations. This finding aligns with recent findings of independence of tonal information and tempo (Schellenberg et al., 2014). In terms of dimensional salience, these data argue for independence, as no interaction emerged despite equalization of (or at least changes to) relative main effect sizes (Prince, 2011).

\section{General Discussion}

Two experiments explored how systematic manipulations of pitch and temporal surface and structural information (contour, rhythm, tonality, and meter) affected similarity ratings of unfamiliar melodies. In both experiments, surface information was the more effective predictor of ratings. Temporal factors were more influential in Experiment 1, possibly because tempo was constant, enabling the use of both absolute and relative timing cues in similarity judgments, whereas only relative pitch information was available by virtue of the use of transposition. In Experiment 2, the tempo of the melodies varied within each trial, transpositions were prepared with chord cadence prefixes, and pitch became the dominant dimension. Neither experiment obtained an interaction between dimensions, and within-dimension interactions between surface and structure were small and unreliable.

This detailed examination of the role of surface and structure in both pitch and time on melodic similarity offers several theoretical

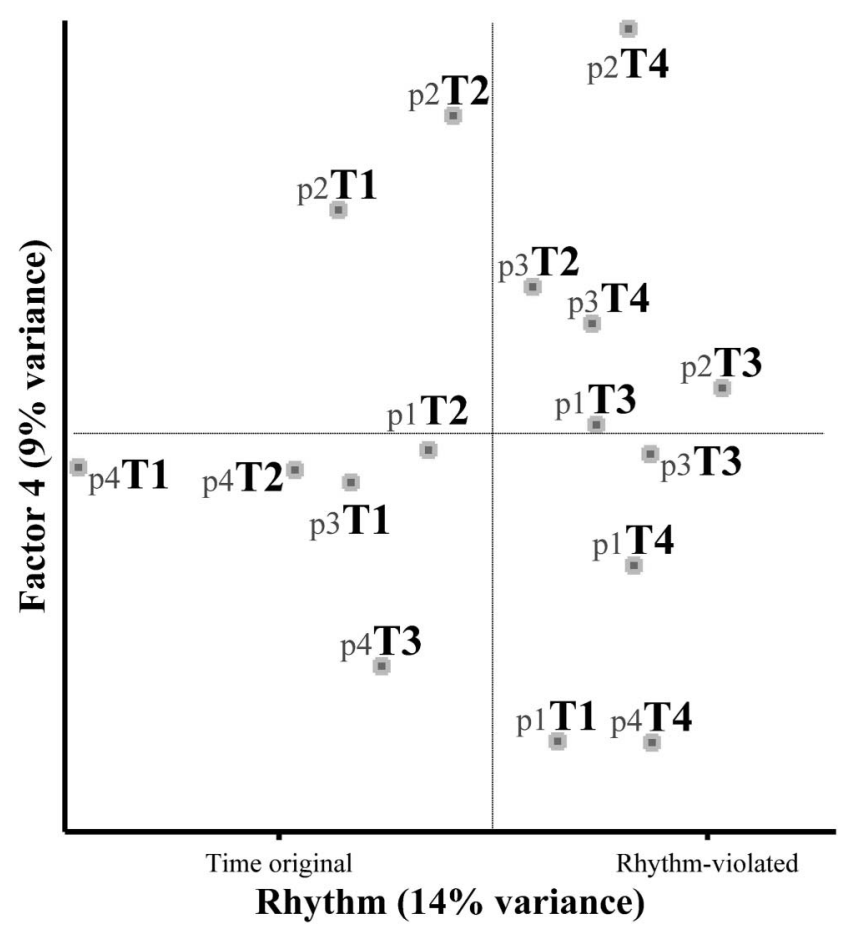

Figure 12. Factors 3 (interpreted as rhythm) and 4 (uninterpreted) of the factor analysis solution of perceived similarity ratings of all compared variants, for Experiment 2. For clarity, data labels emphasize time levels, and internal axes crossing at the origin are added. 
advances. First, the relative strength of pitch and temporal factors is flexible, altered dramatically by changing the tempo and adding a chord prefix. Second, the factorial design revealed independent roles of pitch and time, regardless of the relative strength of these dimensions. Third, interactions between surface and structural information were unreliable and never occurred between dimensions. Subsidiary findings came from the minimal role of expertise and overall effects of melody order.

In Experiment 1, the influence of rhythm and meter on similarity ratings dominated that of contour and tonality. Adding a chord cadence and varying the tempo between melodies (Experiment 2) reversed this bias. This finding that judgments of melodic similarity are flexible is entirely new, as far as the author is aware. One explanation is that listeners rely more on absolute timing information than predicted by the more conventional approach that listeners encode temporal patterns in relative terms instead of absolute (e.g., Drake \& Botte, 1993; Miller \& McAuley, 2005). This is not the only evidence of memory for absolute duration in musical sequences, as Levitin and Cook (1996) found that $72 \%$ of untrained listeners' vocal productions of popular melodies were within $8 \%$ of the actual tempo (ruling out effects of articulatory constraints). Moreover, there is evidence of the importance of absolute information in melodic memory and recognition, such as tempo (Halpern, 1988; Halpern \& Mullensiefen, 2008; Schellenberg et al., 2014), timbre (Lange \& Czernochowski, 2013; PoulinCharronnat et al., 2004), key or pitch height (Creel, 2011; Halpern, 1989; Schellenberg \& Trehub, 2003), and articulation (Wee Hun Lim \& Goh, 2013).

However, the importance of absolute timing information does not explain the change in weighting of contour, rhythm, tonality, and meter-this information was the same in both experiments, so why did their relative importance vary? A possible explanation derives from dimensional salience (Prince, Thompson, et al., 2009), in which the informative value of a dimension can prioritize it at the expense of other dimensions, even when entirely irrelevant to the task. This prioritization affects the dimension as a whole, such that, in this case, all temporal information-including rhythm and meter-became more important when the dimension was more reliable by virtue of preserving the absolute timing relations. Previous work on varying the salience of a dimension has focused on pitch manipulations (Prince, 2014; Prince, Schmuckler, et al., 2009); the current findings suggest that the preservation of tempo can have a similar effect on the salience of time.

The factorial design of the experiments (as recommended by Eerola et al., 2001) enabled stronger evaluations of the relations between manipulated variables. Additionally, the large set size and counterbalancing arrangements vastly reduced the potential role of familiarity with the melodies. In this context, the findings were consistent with complete independence between pitch and time, as there were no significant interactions between these dimensions in either experiment.

The litany of conflicting reports in the literature on pitch-time integration demonstrates that they cannot be purely independent or interactive (for reviews, see Prince, 2011; Schellenberg et al., 2014). Instead, the question of interest is what influences the pattern observed in a given circumstance. One potential factor is whether the nature of the task fosters a global or local style of processing, whereby global processing makes interactive relations more likely (Jones \& Boltz, 1989; Tillmann \& Lebrun-Guillaud,
2006). Melodic similarity is a global task, and yet there were independent contributions of both pitch and time in these data. Preserving the original first and last pitches and durations in all variants was specifically intended to reduce the effectiveness of a local strategy, such as focusing on one section of the melody (e.g., the beginning or end). The stage model of pitch-time interactions proposes that the dimensions are initially separate, and recombined at a later stage in processing (Peretz \& Zatorre, 2005; Thompson, Hall, \& Pressing, 2001). The present data do not fit this approach either-evaluating melodic similarity clearly involves late stages of processing, and yet independence remained.

Event coherence may influence how participants attend to sequences (Jones \& Boltz, 1989). Events are coherent if the pitch accent structure coincides with the temporal accent structure-for instance, ending a melodic phrase (pitch accent) with a lengthened note (temporal accent); this leads to greater integration of pitch and temporal information than if they are offset. Melodies with coherent pitch-time accent structures are recognized (same-different) more accurately than if they are incoherent (Boltz, 1998; Schulkind, 1999). In the present experiments, the coincidence of pitch and temporal accent structures was not systematically controlled, although it almost certainly varied. Thus, perhaps listeners did not adopt an integrated attending style, as only 1/16 of the melodies (the p1t1 variants) would have had a consistently coherent accent structure. Still, if integrating pitch and time is subject to a number of conditions (coherence, task difficulty, task design, etc.), then perhaps it is afforded only by particular contexts rather than the default listening mode.

Attenuating the difference between the main effect sizes of pitch and temporal factors did not uncover an interaction between them (in Experiment 2), suggesting that any potential difference in salience between dimensions (in Experiment 1) was not obscuring an interaction between pitch and time. Research on goodness ratings of single melodies with similar manipulations to the present experiments found that pitch and time interacted when the main effect sizes (squared semipartial correlations) of the two dimensions were more equal (Prince, 2011). That finding was consistent with an interpretation that interactions between dimensions can become obscured when one dimension is more salient than another (Prince, Schmuckler, et al., 2009). Yet in the current experiments, there was no hint of an interaction, even when the effect sizes were nearly identical.

Testing the role of surface and structure in melodic similarity was among the primary foci of the present study. The overall superiority of surface information over structure in similarity ratings of unfamiliar melodies aligns with previous research, but examining exactly how these forms of information combine is unique to this research. Although ANOVA analyses suggested subtle interactions between surface and structure, these occurred only within a given dimension (contour with tonality, and rhythm with meter), and were unreliable and inconsistent (see Tables 3 and 4). No interactions involved both dimensions (such as contour with rhythm), giving further weight to the observed independence of pitch and time. The rhythm-meter interaction was unreliable (only in Experiment 1) and counterintuitive, suggesting that meter was more influential on ratings when the two melodies had a different rhythm. This pattern was inconsistent with the contour-tonality interaction, which was that tonality only contributed to perceived similarity when the contour remained the same in a given melody 
pair. Although this interaction was more consistent across experiment, it emerged only in the ANOVA analysis, just barely reached significance, and only in Experiment 2. Overall, surface and structure were largely independent in these experiments, and particularly so across dimension, which is a novel finding in melodic similarity research.

The present sample was representative of an undergraduate population in that the average experience was around three years of musical training (mode $=0$ ). Expertise had little bearing on the findings, in that greater musical training afforded improved ability to use contour - but not at the expense of other information. It is possible that highly trained musicians would employ a more analytic strategy and would demonstrate greater sensitivity to structural information (Bigand, 1997; Krumhansl \& Shepard, 1979). For instance, Frankland and Cohen (1996) found that a tonal context improved pitch height comparison accuracy for musicians only, but there is also evidence of both populations benefitting from tonality (Schulze, Dowling, \& Tillmann, 2012). In the context of melodic recognition, musicians can also be more flexible in their reliance on tonal or contour information, but moderately experienced listeners also use both types (Dowling, 1986). Musicians use a slightly different brain network for remembering tonal sequences, consistent with a more exact representation of the sequence (Schulze, Mueller, \& Koelsch, 2011). But on the whole, all listeners show remarkably similar perceptual processes and neural activity in response to music (Bigand \& Poulin-Charronnat, 2006; Koelsch, Gunter, Friederici, \& Schröger, 2000), including studies of perceived similarity (Halpern, 1984; Halpern et al., 1998). Moreover, expertise appears not to change the pattern of pitch-time integration (Boltz, 1989; Hébert \& Peretz, 1997; Lebrun-Guillaud \& Tillmann, 2007; Palmer \& Krumhansl, 1987; Smith \& Cuddy, 1989; Tillmann \& Lebrun-Guillaud, 2006). If anything, musicians may be better at separating the dimensions and attending selectively (Pitt \& Monahan, 1987), but given that the current findings already showed independent effects, it is unlikely that increased musical expertise would change the observed pitch-time integration pattern. Addressing this question specifically will require direct comparisons between highly trained and untrained listeners, similar to more general research on the use of surface and structure in physics experts and novices (Chi et al., 1981).

Another potential influence on the results comes from order effects-that is, the same stimuli sometimes received different ratings depending on the order of their presentation. Similarity ratings were lower when the first sequence of a trial adhered more to the original melody's characteristics (e.g., p1t1-p4t1 vs. p4t1p1t1 in Experiment 2; see Figure 8), in agreement with previous findings in both pitch (Bartlett \& Dowling, 1988; Watkins, 1985) and time (Bharucha \& Pryor, 1986; Kidd, Boltz, \& Jones, 1984). Bartlett and Dowling (1988) showed that their order effects were not caused by greater memorability of more tonal melodies, and Schellenberg (2001) explained that "going out-of-tune is more noticeable than going in-tune" (p. 223) when discriminating musical intervals. Both follow from Krumhansl's $(1979,1990)$ formalization of contextual asymmetry-pitches not belonging to the current musical key are judged as more similar to pitches inside the key than vice versa. For both experiments in the present study, order effects were larger for time manipulation levels (Figures 4 and 10) than pitch manipulation levels (Figures 3 and 9). However the same basic patterns emerge on either side of the diagonalsimilarity ratings generally decrease with increasing distance from the diagonal. Overall, the order effects seem to indicate that the task was easier when the less-violated sequence occurred first, but there were no observable qualitative differences in how listeners used and combined the surface and structural information across order.

There are limitations to this research that warrant contemplation. First, all of the seed melodies used only the most common metrical framework of Western music (4/4 time). It is unlikely that the roles of pitch and temporal surface and structure function in a radically different way for other meters (cf. Smith \& Cuddy, 1989), but is untested in a melodic similarity context.

Second, the dichotomous manipulations of tonality and meter (tonal-atonal and metric-ametric) do not capture more nuanced manipulations of tonality and meter, such as contrasting the same pitch sequence in different metrical frameworks. Likewise, the major and minor forms of tonality are perceptually subtle (Halpern, 1984; Halpern et al., 1998) but structurally significant.

Third, the manipulations in the stimuli may not have been as independent as hoped, particularly rhythm and meter. Rearranging the durations of a sequence can affect its metrical strength (Povel $\&$ Essens, 1985); in this case, the randomly reordered $t 3$ variants had lower metricality than $\mathrm{t} 1$, as measured by correlation with the Palmer and Krumhansl (1990) metric hierarchy. However, the present regression analysis used separate and continuous predictors of meter and rhythm, and there were no significant interaction terms; moreover, the factor analysis supported independent roles for these predictors (see Figure 5). Regardless, future work may be able to separate rhythmic and metrical information more effectively.

In closing, the perceived similarity between two novel melodies depends on surface and structural features of pitch and time. For immediate similarity ratings of novel sequences that vary in these properties, surface information is more influential. Within a dimension, surface and structure may show small interactions, but not across dimensions. The relative contribution of pitch and time is flexible based on the availability of relative versus absolute information, but these dimensions function independently regardless of which is stronger.

\section{References}

Abe, J. I., \& Okada, A. (2004). Integration of metrical and tonal organization in melody perception. Japanese Psychological Research, 46, 298-307. doi:10.1111/j.1468-5584.2004.00262.x

Acevedo, S., Temperley, D., \& Pfordresher, P. Q. (2014). Effects of metrical encoding on melody recognition. Music Perception: An Interdisciplinary Journal, 31, 372-386. doi:10.1525/mp.2014.31.4.372

Barnes, R., \& Johnston, H. (2010). The role of timing deviations and target position uncertainty on temporal attending in a serial auditory pitch discrimination task. Quarterly Journal of Experimental Psychology (2006), 63, 341-355. doi:10.1080/17470210902925312

Barnes, R., \& Jones, M. R. (2000). Expectancy, attention, and time. Cognitive Psychology, 41, 254-311. doi:10.1006/cogp.2000.0738

Bartlett, J. C., \& Dowling, W. J. (1988). Scale structure and similarity of melodies. Music Perception, 5, 285-314. doi:10.2307/40285401

Bharucha, J. J., \& Pryor, J. H. (1986). Disrupting the isochrony underlying rhythm: An asymmetry in discrimination. Perception \& Psychophysics, 40, 137-141. doi:10.3758/BF03203008 
Bigand, E. (1997). Perceiving musical stability: The effect of tonal structure, rhythm, and musical expertise. Journal of Experimental Psychology: Human Perception and Performance, 23, 808-822. doi:10.1037/ 0096-1523.23.3.808

Bigand, E., \& Poulin-Charronnat, B. (2006). Are we "experienced listeners"? A review of the musical capacities that do not depend on formal musical training. Cognition, 100, 100-130. doi:10.1016/j.cognition .2005 .11 .007

Boltz, M. G. (1989). Perceiving the end: Effects of tonal relationships on melodic completion. Journal of Experimental Psychology: Human Perception and Performance, 15, 749-761. doi:10.1037/0096-1523.15.4 .749

Boltz, M. G. (1998). The processing of temporal and nontemporal information in the remembering of event durations and musical structure. Journal of Experimental Psychology: Human Perception and Performance, 24, 1087-1104. doi:10.1037/0096-1523.24.4.1087

Boltz, M. G. (2011). Illusory tempo changes due to musical characteristics. Music Perception, 28, 367-386. doi:10.1525/mp.2011.28.4.367

Bregman, A. S. (1990). Auditory scene analysis: The perceptual organization of sound. Cambridge, MA: MIT Press.

Carterette, E. C., Kohl, D. V., \& Pitt, M. A. (1986). Similarities among transformed melodies - the abstraction of invariants. Music Perception, 3, 393-409. doi:10.2307/40285345

Chi, M. T. H., Feltovich, P. J., \& Glaser, R. (1981). Categorization and representation of physics problems by experts and novices. Cognitive Science, 5, 121-152. doi:10.1207/s15516709 $\operatorname{cog} 0502 \_2$

Creel, S. C. (2011). Specific previous experience affects perception of harmony and meter. Journal of Experimental Psychology: Human Perception and Performance, 37, 1512-1526. doi:10.1037/a0023507

Cuddy, L. L., \& Cohen, A. J. (1976). Recognition of transposed melodic sequences. The Quarterly Journal of Experimental Psychology, 28, 255-270. doi:10.1080/14640747608400555

Cuddy, L. L., Cohen, A. J., \& Mewhort, D. J. (1981). Perception of structure in short melodic sequences. Journal of Experimental Psychology: Human Perception and Performance, 7, 869-883. doi:10.1037/ 0096-1523.7.4.869

Desain, P., \& Honing, H. (2003). The formation of rhythmic categories and metric priming. Perception, 32, 341-365. doi:10.1068/p3370

Deutsch, D. (Ed.). (2013). The psychology of music (3rd ed.). San Diego, CA: Academic Press.

Dewitt, L. A., \& Crowder, R. G. (1986). Recognition of novel melodies after brief delays. Music Perception, 3, 259-274. doi:10.2307/40285336

Dowling, W. J. (1973). Rhythmic groups and subjective chunks in memory for melodies. Perception \& Psychophysics, 14, 37-40. doi:10.3758/ BF03198614

Dowling, W. J. (1978). Scale and contour: Two components of a theory of memory for melodies. Psychological Review, 85, 341-354. doi:10.1037/ 0033-295X.85.4.341

Dowling, W. J. (1986). Context effects on melody recognition: Scale-step versus interval representations. Music Perception, 3, 281-296. doi:10.2307/ 40285338

Dowling, W. J. (1991). Tonal strength and melody recognition after long and short delays. Perception \& Psychophysics, 50, 305-313. doi:10.3758/ BF03212222

Dowling, W. J., Bartlett, J. C., Halpern, A. R., \& Andrews, M. W. (2008). Melody recognition at fast and slow tempos: Effects of age, experience, and familiarity. Perception \& Psychophysics, 70, 496-502. doi:10.3758/ PP.70.3.496

Dowling, W. J., \& Fujitani, D. S. (1971). Contour, interval, and pitch recognition in memory for melodies. Journal of the Acoustical Society of America, 49, 524-531. doi:10.1121/1.1912382

Dowling, W. J., Kwak, S., \& Andrews, M. W. (1995). The time course of recognition of novel melodies. Perception \& Psychophysics, 57, 136149. doi: $10.3758 / \mathrm{BF} 03206500$
Drake, C., \& Botte, M.-C. (1993). Tempo sensitivity in auditory sequences: Evidence for a multiple-look model. Perception \& Psychophysics, 54, 277-286. doi:10.3758/BF03205262

Edworthy, J. (1985). Interval and contour in melody processing. Music Perception, 2, 375-388. doi:10.2307/40285305

Eerola, T., Järvinen, T., Louhivuori, J., \& Toiviainen, P. (2001). Statistical features and perceived similarity of folk melodies. Music Perception, 18 275-296. doi:10.1525/mp.2001.18.3.275

Eiting, M. H. (1984). Perceptual similarities between musical motifs Music Perception, 2, 78-94. doi:10.2307/40285283

Essens, P. J. (1986). Hierarchical organization of temporal patterns. Perception \& Psychophysics, 40, 69-73. doi:10.3758/BF03208185

Essens, P. J., \& Povel, D. J. (1985). Metrical and nonmetrical representations of temporal patterns. Perception \& Psychophysics, 37, 1-7. doi: 10.3758/BF03207132

Frankland, B. W., \& Cohen, A. J. (1996). Using the Krumhansl and Schmuckler key-finding algorithm to quantify the effects of tonality in the interpolated-tone pitch-comparison task. Music Perception, 14, $57-$ 83. doi: $10.1525 / \mathrm{mp} .2004 .21 .4 .499$

Freedman, E. G. (1999). The role of diatonicism in the abstraction and representation of contour and interval information. Music Perception, 16, 365-387. doi:10.2307/40285797

Garner, W. R. (1970). Good patterns have few alternatives. American Scientist, 58, 34-42.

Garner, W. R. (1974). The processing of information and structure (Vol. 203). Oxford, UK: Erlbaum.

Garner, W. R., \& Felfoldy, G. L. (1970). Integrality of stimulus dimensions in various types of information processing. Cognitive Psychology, 1, 225-241. doi:10.1016/0010-0285(70)90016-2

Garner, W. R., \& Gottwald, R. L. (1968). Perception and learning of temporal patterns. The Quarterly Journal of Experimental Psychology, 20, 97-109. doi:10.1080/14640746808400137

Halpern, A. R. (1984). Perception of structure in novel music. Memory \& Cognition, 12, 163-170. doi:10.3758/BF03198430

Halpern, A. R. (1988). Perceived and imagined tempos of familiar songs. Music Perception, 6, 193-202. doi:10.2307/40285425

Halpern, A. R. (1989). Memory for the absolute pitch of familiar songs Memory \& Cognition, 17, 572-581. doi:10.3758/BF03197080

Halpern, A. R., Bartlett, J. C., \& Dowling, W. J. (1998). Perception of mode, rhythm and contour in unfamiliar melodies: Effects of age and experience. Music Perception, 15, 335-355. doi:10.2307/40300862

Halpern, A. R., \& Mullensiefen, D. (2008). Effects of timbre and tempo change on memory for music. The Quarterly Journal of Experimental Psychology, 61, 1371-1384. doi:10.1080/17470210701508038

Handel, S., \& Oshinsky, J. S. (1981). The meter of syncopated auditory polyrhythms. Perception \& Psychophysics, 30, 1-9. doi:10.3758/ BF03206130

Hébert, S., \& Peretz, I. (1997). Recognition of music in long-term memory: Are melodic and temporal patterns equal partners? Memory \& Cognition, 25, 518-533. doi:10.3758/BF03201127

Jones, M. R., \& Boltz, M. G. (1989). Dynamic attending and responses to time. Psychological Review, 96, 459-491. doi:10.1037/0033-295X.96 .3 .459

Jones, M. R., \& Ralston, J. T. (1991). Some influences of accent structure on melody recognition. Memory \& Cognition, 19, 8-20. doi:10.3758/ BF03198492

Jones, M. R., Summerell, L., \& Marshburn, E. (1987). Recognizing melodies A dynamic interpretation. The Quarterly Journal of Experimental Psychology A: Human Experimental Psychology, 39, 89-121. doi:10.1080/ 0272498874300005

Kidd, G., Boltz, M. G., \& Jones, M. R. (1984). Some effects of rhythmic context on melody recognition. American Journal of Psychology, 97, 153-173. doi:10.2307/1422592 
Koelsch, S., Gunter, T. C., Friederici, A. D., \& Schröger, E. (2000). Brain indices of music processing: "Nonmusicians" are musical. Journal of Cognitive Neuroscience, 12, 520-541. doi:10.1162/089892900562183

Krumhansl, C. L. (1979). Psychological representation of musical pitch in a tonal context. Cognitive Psychology, 11, 346-374. doi:10.1016/00100285(79)90016-1

Krumhansl, C. L. (1990). Cognitive foundations of musical pitch. New York, NY: Oxford University Press.

Krumhansl, C. L. (1991). Memory for musical surface. Memory \& Cognition, 19, 401-411. doi:10.3758/BF03197145

Krumhansl, C. L. (2000). Rhythm and pitch in music cognition. Psychological Bulletin, 126, 159-179. doi:10.1037/0033-2909.126.1.159

Krumhansl, C. L., \& Cuddy, L. L. (2010). A theory of tonal hierarchies in music. In M. R. Jones, R. R. Fay, \& A. N. Popper (Eds.), Music perception. Springer handbook of auditory research (pp. 57-81). New York, NY: Springer Science. doi:10.1007/978-1-4419-6114-3_3

Krumhansl, C. L., \& Schmuckler, M. A. (1986, July). Key-finding in music: An algorithm based on pattern matching to tonal hierarchies. Paper presented at the 19th Annual Meeting of the Society of Mathematical Psychology, Cambridge, MA.

Krumhansl, C. L., \& Shepard, R. N. (1979). Quantification of the hierarchy of tonal functions within a diatonic context. Journal of Experimental Psychology: Human Perception and Performance, 5, 579-594. doi:10.1037/00961523.5.4.579

Kruskal, J. B., \& Wish, M. (1978). Multidimensional scaling. Beverly Hills, CA: Sage.

Lamont, A., \& Dibben, N. (2001). Motivic structure and the perception of similarity. Music Perception, 18, 245-274. doi:10.1525/mp.2001.18.3 .245

Lange, K., \& Czernochowski, D. (2013). Does this sound familiar? Effects of timbre change on episodic retrieval of novel melodies. Acta Psychologica, 143, 136-145. doi:10.1016/j.actpsy.2013.03.003

Lebrun-Guillaud, G., \& Tillmann, B. (2007). Influence of a tone's tonal function on temporal change detection. Perception \& Psychophysics, 69, 1450-1459. doi:10.3758/BF03192959

Lerdahl, F., \& Jackendoff, R. (1983). A generative theory of tonal music. Cambridge, MA: MIT Press.

Levitin, D. J., \& Cook, P. R. (1996). Memory for musical tempo: Additional evidence that auditory memory is absolute. Perception \& Psychophysics, 58, 927-935. doi:10.3758/BF03205494

Massaro, D. W., Kallman, H. J., \& Kelly, J. L. (1980). The role of tone height, melodic contour, and tone chroma in melody recognition. Journal of Experimental Psychology: Human Learning and Memory, 6, 77-90. doi:10.1037/0278-7393.6.1.77

McAdams, S., Vieillard, S., Houix, O., \& Reynolds, R. (2004). Perception of musical similarity among contemporary thematic materials in two instrumentations. Music Perception, 22, 207-237. doi:10.1525/mp.2004 .22 .2 .207

McAuley, J. D. (2010). Tempo and rhythm. In M. R. Jones, R. R. Fay, \& A. N. Popper (Eds.), Music perception. Springer handbook of auditory research (pp. 165-199). New York, NY: Springer Science + Business Media.

Miller, N. S., \& McAuley, J. D. (2005). Tempo sensitivity in isochronous tone sequences: The multiple-look model revisited. Perception \& Psychophysics, 67, 1150-1160. doi:10.3758/BF03193548

Monahan, C. B., \& Carterette, E. C. (1985). Pitch and duration as determinants of musical space. Music Perception, 3, 1-32. doi:10.2307/ 40285320

Monahan, C. B., Kendall, R. A., \& Carterette, E. C. (1987). The effect of melodic and temporal contour on recognition memory for pitch change. Perception \& Psychophysics, 41, 576-600. doi:10.3758/BF03210491

Motz, B. A., Erickson, M. A., \& Hetrick, W. P. (2013). To the beat of your own drum: Cortical regularization of non-integer ratio rhythms toward metrical patterns. Brain and Cognition, 81, 329-336. doi:10.1016/j .bandc.2013.01.005

Palmer, C., \& Krumhansl, C. L. (1987). Independent temporal and pitch structures in determination of musical phrases. Journal of Experimental Psychology: Human Perception and Performance, 13, 116-126. doi: 10.1037/0096-1523.13.1.116

Palmer, C., \& Krumhansl, C. L. (1990). Mental representations for musical meter. Journal of Experimental Psychology: Human Perception and Performance, 16, 728-741. doi:10.1037/0096-1523.16.4.728

Peretz, I., \& Zatorre, R. J. (2005). Brain organization for music processing. Annual Review of Psychology, 56, 89-114. doi:10.1146/annurev.psych .56 .091103 .070225

Pfordresher, P. Q., \& Mantell, J. T. (2014). Singing with yourself: Evidence for an inverse modeling account of poor-pitch singing. Cognitive Psychology, 70, 31-57. doi:10.1016/j.cogpsych.2013.12.005

Pitt, M. A., \& Monahan, C. B. (1987). The perceived similarity of auditory polyrhythms. Perception \& Psychophysics, 41, 534-546. doi:10.3758/ BF03210488

Pollard-Gott, L. (1983). Emergence of thematic concepts in repeated listening to music. Cognitive Psychology, 15, 66-94. doi:10.1016/00100285(83)90004-X

Poulin-Charronnat, B., Bigand, E., Lalitte, P., Madurell, F., Vieillard, S., \& McAdams, S. (2004). Effects of a change in instrumentation on the recognition of musical materials. Music Perception, 22, 239-263. doi: 10.1525/mp.2004.22.2.239

Povel, D. J., \& Essens, P. (1985). Perception of temporal patterns. Music Perception, 2, 411-440. doi:10.2307/40285311

Prince, J. B. (2011). The integration of stimulus dimensions in the perception of music. Quarterly Journal of Experimental Psychology (2006), 64 2125-2152. doi:10.1080/17470218.2011.573080

Prince, J. B. (2014). Pitch structure, but not selective attention, affects accent weightings in metrical grouping. Journal of Experimental Psychology: Human Perception and Performance. Advance online publication. doi:10.1037/a0037730

Prince, J. B., Schmuckler, M. A., \& Thompson, W. F. (2009). The effect of task and pitch structure on pitch-time interactions in music. Memory \& Cognition, 37, 368-381. doi:10.3758/MC.37.3.368

Prince, J. B., Thompson, W. F., \& Schmuckler, M. A. (2009). Pitch and time, tonality and meter: How do musical dimensions combine? Journal of Experimental Psychology: Human Perception and Performance, 35, 1598-1617. doi: $10.1037 / \mathrm{a} 0016456$

Repp, B. H. (2005). Sensorimotor synchronization: A review of the tapping literature. Psychonomic Bulletin \& Review, 12, 969-992. doi:10.3758/ BF03206433

Repp, B. H., London, J., \& Keller, P. E. (2011). Perception-production relationships and phase correction in synchronization with two-interval rhythms. Psychological Research, 75, 227-242. doi:10.1007/s00426010-0301-8

Repp, B. H., \& Su, Y. H. (2013). Sensorimotor synchronization: A review of recent research (2006-2012). Psychonomic Bulletin \& Review, 20, 403-452. doi:10.3758/s13423-012-0371-2

Rosner, B. S., \& Meyer, L. B. (1986). The perceptual roles of melodic process, contour, and form. Music Perception, 4, 1-39. doi:10.2307/ 40285350

Schellenberg, E. G. (2001). Asymmetries in the discrimination of musical intervals: Going out-of-tune is more noticeable than going in-tune Music Perception, 19, 223-248. doi:10.1525/mp.2001.19.2.223

Schellenberg, E. G., Stalinski, S. M., \& Marks, B. M. (2013). Memory for surface features of unfamiliar melodies: Independent effects of changes in pitch and tempo. Psychological Research, 78, 84-95.

Schellenberg, E. G., \& Trehub, S. E. (2003). Good pitch memory is widespread. Psychological Science, 14, 262-266. doi:10.1111/14679280.03432 
Schmuckler, M. A. (1999). Testing models of melodic contour similarity. Music Perception, 16, 295-326. doi:10.2307/40285795

Schmuckler, M. A. (2004). Pitch and pitch structures. In J. Neuhoff (Ed.), Ecological psychoacoustics (pp. 271-315). San Diego, CA: Elsevier Science.

Schmuckler, M. A. (2009). Components of melodic processing. In S. Hallam, I. Cross, \& M. Thaut (Eds.), Oxford handbook of music psychology (pp. 93-106). Oxford, UK: Oxford University Press.

Schmuckler, M. A. (2010). Melodic contour similarity using folk melodies. Music Perception, 28, 169-193. doi:10.1525/mp.2010.28.2.169

Schulkind, M. D. (1999). Long-term memory for temporal structure: Evidence from the identification of well-known and novel songs. Memory \& Cognition, 27, 896-906. doi:10.3758/BF03198542

Schultz, B. G., Stevens, C. J., Keller, P. E., \& Tillmann, B. (2013). The implicit learning of metrical and nonmetrical temporal patterns. Quarterly Journal of Experimental Psychology (2006), 66, 360-380. doi:10.1080/17470218 .2012 .712146

Schulze, K., Dowling, W. J., \& Tillmann, B. (2012). Working memory for tonal and atonal sequences during a forward and a backward recognition task. Music Perception, 29, 255-267. doi:10.1525/mp.2012.29.3.255

Schulze, K., Mueller, K., \& Koelsch, S. (2011). Neural correlates of strategy use during auditory working memory in musicians and non-musicians. European Journal of Neuroscience, 33, 189-196. doi:10.1111/j.1460-9568 .2010.07470.x

Serafine, M. L., Glassman, N., \& Overbeeke, C. (1989). The cognitive reality of hierarchic structure in music. Music Perception, 6, 397-430. doi: $10.2307 / 40285440$

Smith, K. C., \& Cuddy, L. L. (1989). Effects of metric and harmonic rhythm on the detection of pitch alterations in melodic sequences. Journal of Experimental Psychology: Human Perception and Performance, 15, 457-471. doi:10.1037/0096-1523.15.3.457

Stalinski, S. M., \& Schellenberg, E. G. (2010). Shifting perceptions: Developmental changes in judgments of melodic similarity. Developmental Psychology, 46, 1799-1803. doi:10.1037/a0020658

Thompson, W. F., Hall, M. D., \& Pressing, J. (2001). Illusory conjunctions of pitch and duration in unfamiliar tone sequences. Journal of Experi- mental Psychology: Human Perception and Performance, 27, 128-140. doi:10.1037/0096-1523.27.1.128

Tillmann, B., Gosselin, N., Bigand, E., \& Peretz, I. (2012). Priming paradigm reveals harmonic structure processing in congenital amusia. Cortex: A Journal Devoted to the Study of the Nervous System and Behavior, 48, 1073-1078. doi:10.1016/j.cortex.2012.01.001

Tillmann, B., \& Lebrun-Guillaud, G. (2006). Influence of tonal and temporal expectations on chord processing and on completion judgments of chord sequences. Psychological Research, 70, 345-358. doi:10.1007/ s00426-005-0222-0

Trainor, L. J., McDonald, K. L., \& Alain, C. (2002). Automatic and controlled processing of melodic contour and interval information measured by electrical brain activity. Journal of Cognitive Neuroscience, 14, 430-442. doi:10.1162/089892902317361949

Trehub, S. E., \& Hannon, E. E. (2006). Infant music perception: Domaingeneral or domain-specific mechanisms? Cognition, 100, 73-99. doi 10.1016/j.cognition.2005.11.006

Trehub, S. E., Schellenberg, E. G., \& Kamenetsky, S. B. (1999). Infants' and adults' perception of scale structure. Journal of Experimental Psychology: Human Perception and Performance, 25, 965-975. doi:10.1037/0096-1523 .25 .4 .965

van Egmond, R., \& Povel, D.-J. (1996). Perceived similarity of exact and inexact transpositions. Acta Psychologica, 92, 283-295. doi:10.1016/ 0001-6918(95)00043-7

van Egmond, R., Povel, D.-J., \& Maris, E. (1996). The influence of height and key on the perceptual similarity of transposed melodies. Perception \& Psychophysics, 58, 1252-1259. doi:10.3758/BF03207557

Watkins, A. J. (1985). Scale, key, and contour in the discrimination of tuned and mistuned approximations to melody. Perception \& Psychophysics, 37, 275-285. doi:10.3758/BF03211349

Wee Hun Lim, S. W. H., \& Goh, W. D. (2013). Articulation effects in melody recognition memory. Quarterly Journal of Experimental Psychology (2006), 66, 1774-1792. doi:10.1080/17470218.2013.766758

Received April 30, 2014

Revision received August 22, 2014 Accepted August 28, 2014 